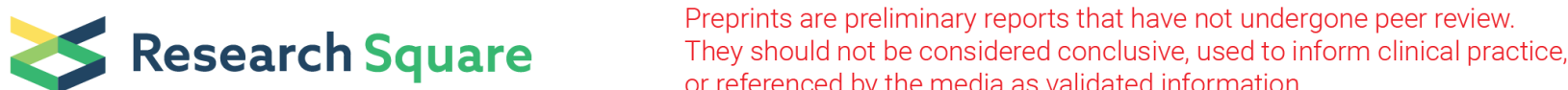

\section{NLR combined with SaO2 predict severe illness among COVID-19 patients: a currently updated model}

Zili Zhang ( $\sim$ zhangzili_04@163.com )

Stata Key Laboratory of Respiratory Diseases, National Clinical Research Center for Respiratory Diseases, Guangzhou Institute of Respiratory Health, the first Affiliated Hospital, Guangzhou Medical University https://orcid.org/0000-0002-8544-2339

Xiansheng Zeng

Xiangyang Central Hospital

Jian Wang

Guangzhou Medical University

E Guo

Xiangyang Central Hospital

Minglong Fu

Hubei University of Medicine

Xuejiao Yang

Xiangyang Central Hospital

Yun Bai

Laohekou First People's Hospital

Qiaoxin Huang

Xiangyang Infectious Disease Hospital

Zhuowei Li

Guangzhou Medical University

Jingyi Xu

Guangzhou Medical University

Yuanyuan Li

Guangzhou Medical University

Xiaodan Zhang

Guangzhou Medical University

Fei Liu

Guangzhou Medical University

Liang Yuan

Guangzhou Medical University

Xiaohui Xie

Guangzhou Medical University

Qiongqiong Li

Guangzhou Medical University

Bingxian Deng

Guangzhou Medical University

\section{Lingzhu Chen}

Guangzhou Medical University

Yongxuan Gao

Guangzhou Medical University

Lan Wang

Guangzhou Medical University

Zhou Cai

Guangzhou Medical University

Zhanbei Zhu

Guangzhou Medical University

Fanjie Lin

Guangzhou Medical University

Wei Liu

Guangzhou Medical University

Hua Guo

Guangzhou Medical University 


\section{Qinghui Huang}

Guangzhou Medical University

Nanshan Zhong

Guangzhou Medical University

Wenju Lu

Guangzhou Medical University

\section{Research}

Keywords: COVID-19, predictive model, NLR, SaO2, Principal Component Analysis

Posted Date: August 26th, 2020

DOI: https://doi.org/10.21203/rs.3.rs-64080/v1

License: @ (i) This work is licensed under a Creative Commons Attribution 4.0 International License. Read Full License 


\section{Abstract}

Objectives: The pandemic of the coronavirus disease 2019 (COVID-19) continuously poses a serious threat to public health, highlighting an urgent need for simple and efficient early detection and prediction.

Methods: We comprehensively investigated and reanalyzed the published indexes and models for predicting severe illness among COVID-19 patients in our dataset, and validated them on an independent dataset.

Results: 696 COVID-19 cases in the discovery stage and 337 patients in the validation stage were involved. The AuROC of neutrophil to lymphocyte ratio (NLR) (0.782) was significantly higher than that of the other 11 independent risk indexes in severe outcome prediction. The combination of NLR and oxygen saturation $\left(\mathrm{SaO}_{2}\right)\left(\mathrm{NLR}+\mathrm{SaO}_{2}\right)$ showed the biggest AuROC calculations with a value of 0.901 ; with a cut-off value of 0.532 , it exhibited $84.2 \%$ sensitivity, $88.4 \%$ specificity and $86.8 \%$ correct classification ratio. Moreover, we first identified that principal component analysis (PCA) is an effective tool to predict the severity of COVID-19. We obtained $86.5 \%$ prediction accuracy with $86 \%$ sensitivity when PCA was applied to predict severe illness. In addition, to evaluate the performance of $\mathrm{NLR}+\mathrm{SaO}_{2}$ and $\mathrm{PCA}$, we compared them with currently published predictive models in the same dataset.

Conclusions: It showed that $\mathrm{NLR}+\mathrm{SaO}_{2}$ is an appropriate and promising method for predicting severe illness, followed by PCA. We then validated the results on an independent dataset and revealed that they remained robust accuracy in outcome prediction. This study is significant for early treatment, intervention, triage and saving limited resources.

\section{Background}

Coronavirus disease 2019 (COVID-19) is an unprecedented global threat caused by severe acute respiratory syndrome coronavirus 2 (SARS-CoV-2). Currently, it is rapidly spreading around the world. There are over $13,808,626$ confirmed COVID-19 cases and about 589,978 deaths (up to July 17,2020 ). Over $19 \%$ of COVID-19 patients with mild symptoms may quickly progress to severe or critical conditions ${ }^{1}$. The 28 -day mortality rate was over $60 \%$ in critically ill COVID-19 patients, posing a great pressure on treatment ${ }^{2}$. However, there is no efficient indicators currently available to predict severe illness and progression among COVID-19 patients. Although a number of prediction models have been established, none of them has been validated by independent external studies ${ }^{3}$.

In this retrospective study, we involved 696 COVID-19 cases in the discovery stage and 337 patients in the validation stage, and reanalyzed the expression profiles of the available clinical features and laboratory test parameters in our dataset. The objectives of this study include: Firstly, to validate the published indexes and establish a new simple and efficient combined indexes for assessing the severity of COVID-19; Secondly, to verify and update currently available prediction models.

Principal Component Analysis (PCA) is a mathematical construct that extracts relevant variables from complex datasets into uncorrelated eigen-modes, or "principal component" information by an orthogonal transformation 4 . It is an exploratory tool favored to reduce redundancy in multi-attributes and reveal a simplified structure of disease. To date, the predictive value of PCA has not been tested in patients with COVID-19. In this study, the potential of PCA in outcome prediction of disease would be evaluated.

Last, we assessed the performance of the new combined indexes as well PCA model, compared them with other published predictive models in the same dataset, and also validated them on an independent dataset, with the aim to figure out an efficient early detection and prediction model for evaluating severe illness of COVID-19 patients by comprehensively investigating and assessing the available indexes and models.

\section{Methods}

\section{Study participants}

This retrospective, multi-center studies were conducted at the Xiangyang Central Hospital, Laohekou First People's Hospital, Xiangyang Infectious Disease Hospital, Xiangyang First People's Hospital in Hubei province, and the First Affiliated Hospital of Guangzhou Medical University in Guangdong province between January 30, 2020 and June 30, 2020. In this study, all participants were hospitalized COVID-19 patients ( $N=1033)$, who were confirmed to have SARS-CoV-2 infection by virus nucleic acid test, including 418 mild to moderate cases and 278 severe to critical illness in the discovery stage, and 256 mild to moderate cases and 81 severe to critical in the validation stage. The confirmation and classification of the patients defined according to the Chinese National Health Commission Guidelines for Diagnosis and Treatment of SARS-CoV-2 infection (seventh version) ${ }^{5}$.

\section{Literature search and Selection criteria}

Systematic searches of the literature were performed by using PubMed and Embase through Ovid, bioRxiv, medRxiv, and arXiv (until July 15, 2020). The keywords for the searches included "novel coronavirus" or "nCoV-2019" or "2019-nCoV" or "COVID-19" or "SARS-CoV-2" concatenated with severe prediction models or biomarkers. All eligible articles were retrieved, and their references were searched for further potentially relevant articles. English-or Chinese-language publications reporting data on indexes identified in COVID-19 patients were included if they met the criteria below: 1) patients could be divided into the mild, moderate, severe and critical groups; 2 ) indexes involved were available in the present study. Studies were excluded if comparable indexes were not available or they did not fulfill the inclusion criteria. Finally, 27 studies relevant with biomarkers and more than 10 researches associated with predictive models were included after a close examination $3,6-20$

Data Collection 
Data regarding epidemiological characteristics, clinical, laboratory test and radiological findings were obtained from electronic and paper medical records. Hospitalization admission time, hospital discharge time, and time from illness onset to hospitalization and disease severity on admission were also recorded. Body temperature was measured by an infrared thermometer, and fever was defined as temperature $\geq 37.3^{\circ} \mathrm{C}$. Two researchers independently reviewed the data collection forms to double-check the collected data. All the procedures were approved by the ethics commission of the First Affiliated Hospital of Guangzhou Medical University (Institutional Review Board: 202092) and was performed in compliance with the Declaration of Helsinki. Verbal informed consent were obtained from all participants because the family members were in quarantine.

\section{Principal Component Analysis (pca)}

PCA is a data reduction technique that can convert a large set of variables into a smaller set that still contains most of the original information ${ }^{4}$. Principal components were extracted as a linear combination of the variables. This variance was then removed and a second linear combination built, which iteratively explains the maximum proportion of the whole information. This is called the principal axis method, which leads to orthogonal (uncorrelated) factors. Furthermore, it involves the computation of eigenvalues and eigenvectors of covariance matrices, then these eigenvectors sorted in the descending order of their eigenvalues, and the actual data followed ${ }^{21}$.

\section{Statistical analysis}

Categorical parameters were presented as number (\%) by Chi-squared test, fisher's exact test or Mann-Whitney U test. Normally or non-normally distributed continuous variables were presented as mean (standard deviation, SD) or as median (interquartile range, IQR) by independent $t$ test or Mann-Whitney test, respectively. The Spearman rank correlation coefficient was conducted for the correlation analysis between the selected indexes. Multivariate logistical regression was performed to validate independent risk factors for the severity of COVID-19. Moreover, the combined values for the prediction of disease severity were calculated by binary logistic regression. The accuracy of each independent or combined indexes was determined by AuROC. The optimal threshold value was obtained by calculating the correct classification ratio (CCR). PCA seeks a linear combination of variables such that the principal components (PC) can be extracted. Loadings from the first and second PCs were used to form the weighted component scores ( $\mathrm{Y} 1, \mathrm{Y} 2)$ as a linear combination of the original 12 variables for each participant. Eigen equations showed by $\mathrm{Y} 1, \mathrm{Y} 2$ are combined to indicate a composite disease expression score $\left(Y_{3}=\left|Y_{1} \lambda_{1}\right|+\left|Y_{2} \lambda_{2}\right|\right)$ where $\lambda_{i}$ is the variance explained by each PC (eigenvalue) that accounts for most of the variation. All analyses were done with Stata 15 (StataCorp LLC, TX77845 USA) and OriginLab 2020b software (OriginLab Corporation, MA, USA), using two-sided p values. Statistical significance was set at $p<0.05$.

\section{Results}

\section{Demographic data and baseline characteristics of COVID-19 patients}

The demographic data and baseline characteristics of COVID-19 patients are shown in Table 1. A total of 696 COVID-19 patients (399 males and 297 females) with a average age of 57.8 years, were enrolled in the discovery study. Compared with mild-to-moderate cases, most of the severe-to-critical ill patients were more than 60 years $\left(64.4 \%\right.$ ) and male (64.4\%) with a average age of 62.7 years. $\mathrm{SaO}_{2}<94$ were greatly decreased in severe-to-critical patients (82\%) compared to mild-to-moderate patients. In severe-to-critical group, the median time from illness onset to hospital admission was 10 days (9.0 10.0) and time from illness onset to end hospitalization was 24 days (22.0 27.0), and the above median duration was 8.5 days and 21 days in mild-to-moderate group, respectively. Complications occurred more frequently in severe-to-critical ill patients (69.1\%), with hypertension being the most common comorbidity. The most common symptoms on hospital admission was polypnea in severe-to-critical cases compared to mild-to-moderate patients. The above demographic data and baseline characteristics of COVID-19 patients showed nearly similar trends in the validation study. 
Demographic and baseline of COVID-19 patients on hospital admission.

\begin{tabular}{|c|c|c|c|c|c|c|c|c|}
\hline & \multicolumn{4}{|c|}{ Discovery dataset } & \multicolumn{4}{|c|}{ Validation dataset } \\
\hline & Total & Mild/Moderate & Severe/Critical & $\begin{array}{l}\mathrm{p} \\
\text { value }\end{array}$ & Total & Mild/Moderate & Severe/Critical & $\begin{array}{l}\mathrm{p} \\
\text { value }\end{array}$ \\
\hline & $(n=696)$ & $(n=418)$ & $(n=278)$ & & $(n=337)$ & $(n=256$ & $(n=81)$ & \\
\hline \multicolumn{9}{|l|}{ Demographics } \\
\hline Age, years & $57.8 \pm 14.3$ & $54.5 \pm 14.2$ & $62.7 \pm 13.2$ & $\hat{0} .001$ & $48.4 \pm 16.0$ & $45.4 \pm 14.8$ & $58.5 \pm 16.0$ & $\hat{0.001}$ \\
\hline Age $>60$ years & $323(46.4)$ & 144(34.5) & $179(64.4)$ & $\hat{0} .001$ & $76(23.3)$ & $37(14.7)$ & $39(52.0)$ & $\hat{0.001}$ \\
\hline Male & $399(57.3)$ & $220(52.6)$ & $179(64.4)$ & 0.002 & $172(51.0)$ & $120(46.9)$ & $52(64.2)$ & 0.007 \\
\hline Ever smoker & $42(6.0)$ & $21(5.0)$ & $21(7.6)$ & 0.17 & $21(6.2)$ & $13(5.1)$ & $8(9.9)$ & 0.12 \\
\hline ICU & $158(22.7)$ & $27(6.5)$ & $131(47.1)$ & $\hat{0} .001$ & $47(14.0)$ & $3(1.2)$ & $44(55.7)$ & $\hat{0.001}$ \\
\hline Respiratory rate & $22.3 \pm 4.1$ & $21.2 \pm 2.8$ & $23.9 \pm 5.2$ & $\begin{array}{l}<.001 \\
0.01\end{array}$ & $20.1 \pm 2.7$ & $19.9 \pm 2.6$ & $20.7 \pm 2.8$ & 0.02 \\
\hline$>24$ breaths per min & $154(22.1)$ & $56(13.4)$ & $98(35.3)$ & $\hat{0} .001$ & $14(4.2)$ & $8(3.1)$ & $6(7.4)$ & 0.09 \\
\hline Heart rate & $87.4 \pm 12.2$ & $86.2 \pm 10.6$ & $89.3 \pm 14.2$ & 0.02 & $86.1 \pm 12.3$ & $86.0 \pm 12.3$ & $86.1 \pm 12.5$ & 0.96 \\
\hline$\geq 125$ beats per $\min$ & $5(0.7)$ & $1(0.2)$ & $4(1.4)$ & 0.07 & $8(2.7)$ & $3(1.2)$ & $4(4.9)$ & 0.04 \\
\hline $\mathrm{SaO}_{2}, \%$ & $92.7 \pm 7.8$ & $96.2 \pm 2.6$ & $87.3 \pm 9.7$ & $\hat{0} .001$ & $93.6 \pm 8.4$ & $96.2 \pm 2.7$ & $85.4 \pm 13.4$ & $\hat{0.001}$ \\
\hline$<94$ & $267(38.4)$ & $39(9.3)$ & $228(82.0)$ & $\hat{0} .001$ & $86(25.5)$ & $27(10.6)$ & $59(72.8)$ & $\hat{0} .001$ \\
\hline $\mathrm{O}_{2}$ pressure & $9.4(8.9-9.9)$ & $\begin{array}{l}10.0(9.5- \\
10.6)\end{array}$ & $8.0(7.1-9.0)$ & $\hat{0} .001$ & $\begin{array}{l}9.5(7.6- \\
11.8)\end{array}$ & $9.8(8.6-11.8)$ & $7.9(6.7-11.9)$ & 0.02 \\
\hline $\mathrm{O}_{2}$ concentration & $0.22 \pm 0.06$ & $0.22 \pm 0.05$ & $0.23 \pm 0.07$ & 0.21 & $0.22 \pm 0.04$ & $0.22 \pm 0.04$ & $0.22 \pm 0.03$ & 0.74 \\
\hline $\begin{array}{l}\text { Time from illness onset } \\
\text { to }\end{array}$ & $9.0(9.0-10.0)$ & $8.5(8.0-9.0)$ & $\begin{array}{l}10.0(9.0- \\
10.0)\end{array}$ & $\hat{0} .001$ & $5(3-7)$ & $5(3-7)$ & $5(3-8)$ & 0.11 \\
\hline \multicolumn{9}{|l|}{ hospital admission, days } \\
\hline $\begin{array}{l}\text { Time from illness onset } \\
\text { to }\end{array}$ & $\begin{array}{l}22.0(21.0- \\
23.0)\end{array}$ & $\begin{array}{l}21.0(20.0- \\
23.0)\end{array}$ & $\begin{array}{l}24.0(22.0- \\
27.0)\end{array}$ & 0.007 & $22(12-30)$ & $21(11-38)$ & $32(22-41)$ & $\hat{0.001}$ \\
\hline \multicolumn{9}{|l|}{ end hospitalization, days } \\
\hline \multicolumn{9}{|l|}{ Clinical characteristics } \\
\hline $\begin{array}{l}\text { Fever (temperature } \geq \\
37.3^{\circ} \mathrm{C} \text { ) }\end{array}$ & 649(93.3) & $383(91.6)$ & $266(95.7)$ & 0.037 & $91(27.0)$ & $69(27.0)$ & $22(27.2)$ & 0.97 \\
\hline Highest temperature $\left({ }^{\circ} \mathrm{C}\right)$ & $38.6 \pm 0.65$ & $38.7 \pm 0.64$ & $38.6 \pm 0.68$ & 0.11 & $37.1 \pm 0.74$ & $37.1 \pm 0.72$ & $37.1 \pm 0.81$ & 0.96 \\
\hline Cough & $531(76.3)$ & 292(69.9) & $239(86.0)$ & $\hat{0.001}$ & $220(65.3)$ & $170(66.4)$ & $50(61.7)$ & 0.44 \\
\hline Sputum & $214(30.8)$ & $116(27.8)$ & $98(35.3)$ & 0.036 & $107(31.8)$ & 77(30.1) & $30(37.0)$ & 0.24 \\
\hline Myalgia & $80(11.5)$ & $51(12.2)$ & $29(10.4)$ & 0.47 & $54(16.0)$ & $45(17.6)$ & $9(11.1)$ & 0.17 \\
\hline Fatigue & 235(33.8) & 149(35.7) & $86(30.9)$ & 0.20 & $94(27.9)$ & $70(27.3)$ & $24(29.6)$ & 0.69 \\
\hline Diarrhoea & $20(2.9)$ & 15(3.6) & $5(1.8)$ & 0.17 & $21(6.2)$ & $10(3.9)$ & 11(13.6) & 0.002 \\
\hline Vomiting & $25(3.6)$ & $11(2.6)$ & $14(5.0)$ & 0.10 & $8(2.4)$ & $3(1.2)$ & $5(6.2)$ & 0.01 \\
\hline Rhinobyon & $17(2.4)$ & $7(1.7)$ & 10(3.6) & 0.11 & $9(2.7)$ & $9(3.5)$ & 0 & 0.09 \\
\hline Hemoptysis & $10(1.4)$ & $5(1.2)$ & $5(1.8)$ & 0.51 & $2(0.6)$ & 0 & $2(2.5)$ & 0.01 \\
\hline Headache & $44(6.3)$ & $34(8.1)$ & $10(3.6)$ & 0.016 & $41(12.2)$ & $36(14.1)$ & $5(6.2)$ & 0.06 \\
\hline Sorethroat & $24(3.5)$ & 15(3.6) & $9(3.2)$ & 0.80 & $44(13.1)$ & $36(14.1)$ & $8(9.9)$ & 0.33 \\
\hline
\end{tabular}




\begin{tabular}{|c|c|c|c|c|c|c|c|c|}
\hline \multirow[b]{2}{*}{ Polypnea } & \multicolumn{4}{|c|}{ Discovery dataset } & \multicolumn{4}{|c|}{ Validation dataset } \\
\hline & $322(46.3)$ & $143(34.2)$ & $179(64.4)$ & $\hat{0.001}$ & $51(15.1)$ & $26(10.2)$ & 25(30.9) & $\hat{0.001}$ \\
\hline Shiver & $59(8.5)$ & $37(8.9)$ & $22(7.9)$ & 0.66 & $16(4.8)$ & $13(5.1)$ & $3(3.7)$ & 0.61 \\
\hline Comorbidity & $284(55.2)$ & $130(45.9)$ & 154(69.1) & <. 001 & $122(36.2)$ & $71(27.7)$ & $51(63.0)$ & $\begin{array}{l}<.001 \\
0.001\end{array}$ \\
\hline Hypertension & 196(28.1) & $68(34.9)$ & $128(54.0)$ & <. 0.001 & 78(23.2) & $42(16.4)$ & $36(44.4)$ & $\begin{array}{l}<.001 \\
0.00\end{array}$ \\
\hline Diabetes & $96(13.8)$ & $52(12.4)$ & $44(15.8)$ & 0.20 & $22(6.5)$ & $11(4.3)$ & 11(13.6) & 0.003 \\
\hline Coronary heart disease & $61(8.8)$ & $23(5.5)$ & 38(13.7) & <. 001 & $19(5.6)$ & $9(3.5)$ & $10(12.4)$ & 0.003 \\
\hline Cerebrovascular disease & $27(3.9)$ & $7(1.7)$ & $20(7.2)$ & <. 001 & $6(1.8)$ & $2(0.8)$ & $4(4.9)$ & 0.01 \\
\hline Others & 95(13.7) & $49(11.7)$ & $46(16.6)$ & 0.07 & $50(14.8)$ & $29(11.3)$ & $21(25.9)$ & 0.001 \\
\hline
\end{tabular}

\section{Correlation Analysis Of The Published Variables}

Spearman rank coefficient correlation analysis were done to analyze the association between all selected variables. Heatmap of the correlation matrix showed that correlations between these variables were existed regardless of the outcomes in both discovery (Fig. 1A-D) and validation studies (Fig. 1E-F). For instance, the results showed that neutrophils expression level was highly and positively correlated with leukocytes in mild-to-moderate cases on hospital admission. While when at end hospitalization, we observed a highly and negatively association between albumin and leukocyte, neutrophils, SAA, CRP, LDH (circle size and color indicated relevant strength, dark red and big circles denoted the positively and strong associations, light red and small circles denoted the positively and weak associations). The situation revealed identical characteristic in the validation stage. Correlation analysis showed interaction and co-collinearity were existed between the available variables, and therefore it was improper to predict the severity of COVID-19 by a traditional statistical method.

\section{Dynamic Changes Or Expression Levels Of Laboratory Parameters}

Figure 2a-r visualized dynamic changes of laboratory parameters in mild to moderate and severe-to-critically ill patients during progression of COVID-19 throughout the clinical course (from hospital admission to end hospitalization) in the discovery stage (as well showed in Table 2). Six indexes, including leukocytes, neutrophils, NLR, LDH, CRP and SAA showed a significant continuous downward trend in severe-to-critical cases, but present an almost identical results in mild-to-moderate except SAA. For lymphocytes, eosinophils, basophils, high-sensitivity cardiac troponin I, prothrombin time, D-dimer, serum ferritin, IL-6 and albumin showed a continuous upward effect in severe-to-critical patients and majority of mild-to-moderate also present a similar result. On hospital admission, regardless of the severity or the outcome, majority of patients present an identical monocytes and platelets, but increased significantly afterwards for platelets in mild to moderate. Whereas severe-to-critical cases started with a high-level and dropped significantly afterwards in PLR, and the situation was almost opposite for PCT. 
Table 2

Laboratory findings of COVID-19 patients on hospital admission and end hospitalization.

\begin{tabular}{|c|c|c|c|c|c|c|c|c|}
\hline & \multicolumn{6}{|c|}{ Discovery dataset } & \multirow{2}{*}{\multicolumn{2}{|c|}{$\begin{array}{l}\text { Validation dataset } \\
\text { Hospital admission }\end{array}$}} \\
\hline & \multicolumn{3}{|c|}{ Hospital admission } & \multicolumn{3}{|c|}{ End hospitalization } & & \\
\hline & Total & Mild/Moderate & Severe/Critical & Total & Mild/Moderate & Severe/Critical & Total & Mild/Moderate \\
\hline & $(n=652)$ & $(n=404)$ & $(n=248)$ & $(n=580)$ & $(n=356)$ & $(n=224)$ & $(n=337)$ & $(n=256$ \\
\hline \multirow{2}{*}{$\begin{array}{l}\text { Leukocytes, } \\
\times 10 \bigotimes / L\end{array}$} & 6.17 & 5.4 & $8.25^{\ddagger}$ & 6.02 & 5.69 & $7.05^{\ddagger}$ & 5.92 & 5.48 \\
\hline & $\begin{array}{l}(4.34- \\
9.11)\end{array}$ & $(4.04-7.61)$ & $(5.34-11.59)$ & $\begin{array}{l}(4.72- \\
8.58)\end{array}$ & $(4.57-7.42)$ & $(4.98-13.12)$ & $\begin{array}{l}(4.25- \\
8.50)\end{array}$ & $(4.03-7.57)$ \\
\hline$<4$, or $>10$ & $261(40.0)$ & 135(33.4) & $126(50.8)^{\ddagger}$ & $176(52.2)$ & $83(23.3)$ & $93(41.5)^{\ddagger}$ & $129(38.3)$ & $88(34.4)$ \\
\hline \multirow{2}{*}{$\begin{array}{l}\text { Neutrophils, } \\
\times 10 \otimes / L\end{array}$} & 4.75 & 3.83 & $7.47^{\ddagger}$ & 4.06 & 3.64 & $4.88^{\ddagger}$ & 4.44 & 3.77 \\
\hline & $\begin{array}{l}(2.92- \\
7.75)\end{array}$ & $(2.54-5.93)$ & $(4.11-10.81)$ & $\begin{array}{l}(2.86- \\
6.54)\end{array}$ & $(2.61-5.16)$ & $(3.35-11.25)$ & $\begin{array}{l}(2.83- \\
6.93)\end{array}$ & $(2.41-5.95)$ \\
\hline$<1.8$, or $>6.3$ & $277(47.4)$ & $124(30.7)$ & $153(61.6)^{\ddagger}$ & 181(53.7) & $79(22.2)$ & $102(45.5)^{\ddagger}$ & 134(39.8) & $85(33.2)$ \\
\hline \multirow{2}{*}{$\begin{array}{l}\text { Lymphocytes, } \\
\times 10 \varangle / L\end{array}$} & 0.86 & 1.01 & $0.68^{\ddagger}$ & 1.25 & 1.4 & $1.0^{\ddagger}$ & 0.97 & 1.06 \\
\hline & $\begin{array}{l}(0.6- \\
1.22)\end{array}$ & $(0.72-1.37)$ & $(0.49-0.92)$ & $\begin{array}{l}(0.88- \\
1.69)\end{array}$ & $(1.01-1.77)$ & $(0.7-1.4)$ & $\begin{array}{l}(0.67- \\
1.33)\end{array}$ & $(0.73-1.45)$ \\
\hline$<1.1$ & $447(68.6)$ & $231(57.3)$ & $216(87.3)^{\ddagger}$ & $234(69.4)$ & $110(30.9)$ & $124(55.4)^{\ddagger}$ & $204(60.5)$ & $136(53.1)$ \\
\hline \multirow{2}{*}{$\begin{array}{l}\text { Monocytes, } \times \\
\text { 10区/L }\end{array}$} & 0.34 & 0.34 & 0.33 & 0.42 & 0.42 & 0.45 & 0.35 & 0.35 \\
\hline & $\begin{array}{l}(0.23- \\
0.47)\end{array}$ & $(0.23-0.47)$ & $(0.22-0.49)$ & $\begin{array}{l}(0.31- \\
0.54)\end{array}$ & $(0.32-0.53)$ & $(0.29-0.57)$ & $\begin{array}{l}(0.24- \\
0.47)\end{array}$ & $(0.24-0.46)$ \\
\hline$<0.1$ & 25(3.8) & $11(2.8)$ & $14(5.8)$ & $15(4.5)$ & $5(1.4)$ & $10(4.5)^{\dagger}$ & 12(3.6) & $8(3.1)$ \\
\hline \multirow[t]{2}{*}{$\begin{array}{l}\text { Eosinophils, } \\
\times 10 \otimes / L\end{array}$} & 0.01 & 0.01 & $0^{\ddagger}$ & 0.07 & 0.08 & $0.05^{\ddagger}$ & 0.01 & 0.01 \\
\hline & $(0-0.04)$ & $(0-0.04)$ & $(0-0.02)$ & $\begin{array}{l}(0.03- \\
0.13)\end{array}$ & $(0.04-0.13)$ & $(0.01-0.11)$ & $\begin{array}{l}(0.00- \\
0.04)\end{array}$ & $(0.00-0.04)$ \\
\hline$<0.03$ & $279(42.8)$ & 154(38.1) & $125(50.5)^{\ddagger}$ & $169(50.1)$ & 71(19.9) & $98(43.8)^{\ddagger}$ & $252(74.8)$ & 181(70.7) \\
\hline \multirow[t]{2}{*}{$\begin{array}{l}\text { Basophils, } \times \\
\text { 10囚/L }\end{array}$} & 0.01 & 0.01 & $0.02^{\ddagger}$ & 0.02 & 0.02 & $0.03^{\ddagger}$ & 0.01 & 0.01 \\
\hline & $\begin{array}{l}(0.01- \\
0.02)\end{array}$ & $(0.01-0.02)$ & $(0.01-0.02)$ & $\begin{array}{l}(0.01- \\
0.04)\end{array}$ & $(0.01-0.03)$ & $(0.01-0.04)$ & $\begin{array}{l}(0.01- \\
0.02)\end{array}$ & $(0.01-0.02)$ \\
\hline$<0.02$ & $309(47.4)$ & $224(55.4)$ & $85(34.2)^{\ddagger}$ & $327(97.0)$ & $217(61.0)$ & $110(49.1)^{\ddagger}$ & $285(84.6)$ & $224(87.5)$ \\
\hline \multirow{2}{*}{$\begin{array}{l}\text { Platelets, } \times \\
\text { 10ष/L }\end{array}$} & 200 & 201 & 200 & 226 & 244 & $192^{\ddagger}$ & 199 & 198 \\
\hline & $\begin{array}{l}(145- \\
263)\end{array}$ & $(155-263)$ & $(127-265)$ & $(151-298)$ & $(178-307)$ & $(92-262)$ & $\begin{array}{l}(154- \\
265)\end{array}$ & $(155-266)$ \\
\hline$<125$ & $98(15.2)$ & $39(9.8)$ & $59(24.0)^{\ddagger}$ & 107(31.8) & $37(10.4)$ & $70(31.3)^{\ddagger}$ & $38(11.3)$ & $24(9.4)$ \\
\hline \multirow[t]{2}{*}{$\mathrm{NLR}^{\mathrm{a}}$} & 5.19 & 3.56 & $10.23^{\ddagger}$ & 3.19 & 2.7 & $5.61^{\ddagger}$ & 4.38 & 3.39 \\
\hline & $\begin{array}{l}(2.71- \\
10.53)\end{array}$ & $(2.19-6.71)$ & $(5.24-19.02)$ & $\begin{array}{l}(1.92- \\
6.95)\end{array}$ & $(1.65-4.24)$ & $(2.53-14.3)$ & $\begin{array}{l}(2.33- \\
9.53)\end{array}$ & $(2.05-6.73)$ \\
\hline$>5$ & $325(51)$ & 142(35.8) & $183(76.3)^{\ddagger}$ & $185(54.9)$ & $72(20.2)$ & $113(50.4)^{\ddagger}$ & $152(45.1)$ & $90(35.2)$ \\
\hline PLR $^{b}$ & 222.9 & 204.1 & $291.1^{\ddagger}$ & 175.9 & 174.8 & 179.4 & 208 & 197 \\
\hline
\end{tabular}

Data are median $\left(\mathrm{P}_{25}-\mathrm{P}_{75}\right)$, mean $\pm \mathrm{SD}, \mathrm{n}(\%)$, or $\mathrm{n} / \mathrm{N}(\%)$. $P$ values were calculated by Mann-Whitney $\mathrm{U}$ test, $\mathrm{t}$ test, $\chi^{2}$ test, or Fisher's exact test, as appropriate.

a NLR: Neutrophils to Lymphocytes Ratio; ${ }^{b}$ PLR: Platelets to Lymphocytes Ratio; ${ }^{c}$ LDH: Lactate dehydrogenase; ${ }^{d}$ PCT: Procalcitonin; ${ }^{\text {e }}$ SAA: Serum amyloid $/$

$+P<0.05,{ }^{\ddagger} P<0.01$. 


\begin{tabular}{|c|c|c|c|c|c|c|c|c|}
\hline & \multicolumn{6}{|c|}{ Discovery dataset } & \multicolumn{2}{|c|}{ Validation dataset } \\
\hline & $\begin{array}{l}(151.5- \\
334.7)\end{array}$ & $(143.4-286.3)$ & $(186.5-410.4)$ & $\begin{array}{l}(125- \\
246.5)\end{array}$ & $(128.2-235.4)$ & $(109.0-278.4)$ & $\begin{array}{l}(1484- \\
310)\end{array}$ & $(143-275)$ \\
\hline$>300$ & 203(31.7) & $89(22.4)$ & $114(47.1)^{\ddagger}$ & $92(27.3)$ & $45(12.6)$ & $47(21.0)^{\ddagger}$ & $91(27.0)$ & $51(19.9)$ \\
\hline \multirow[t]{2}{*}{$\mathrm{LDH}^{\mathrm{c}}, \mathrm{U} / \mathrm{L}$} & 314 & 272 & $415^{\ddagger}$ & 238 & 218 & $298^{\ddagger}$ & 232 & 221 \\
\hline & $\begin{array}{l}(234.5- \\
429)\end{array}$ & $(214.8-344.3)$ & $(312-548)$ & $\begin{array}{l}(194- \\
314.5)\end{array}$ & $(186.5-262)$ & $(220-541)$ & $\begin{array}{l}(189- \\
290)\end{array}$ & $(184-266)$ \\
\hline$>245$ & $453(69.5)$ & $235(58.3)$ & $218(87.9)^{\ddagger}$ & $258(76.6)$ & 119(33.4) & $139(62.1)^{\ddagger}$ & $141(41.8)$ & $87(34.0)$ \\
\hline $\begin{array}{l}\text { High- } \\
\text { sensitivity } \\
\text { cardiac }\end{array}$ & 4.8 & 3.6 & $8.7^{\ddagger}$ & 21.3 & 3.9 & $38.5^{\ddagger}$ & 3.50 & 3.20 \\
\hline $\begin{array}{l}\text { troponin I, } \\
\mathrm{pg} / \mathrm{mL}\end{array}$ & $\begin{array}{l}(1.8- \\
14.35)\end{array}$ & $(1.1-8.6)$ & $(3-35.9)$ & $\begin{array}{l}(2.75- \\
125.8)\end{array}$ & $(0.98-36.5)$ & $(4.78-294.03)$ & $\begin{array}{l}(1.00- \\
9.30)\end{array}$ & $(0.70-7.08)$ \\
\hline$>28$ & 103(15.8) & $26(6.3)$ & $77(31.1)^{\ddagger}$ & $58(17.2)$ & $27(7.6)$ & $31(13.8)^{\dagger}$ & $35(10.4)$ & $16(6.3)$ \\
\hline \multirow{2}{*}{$\begin{array}{l}\text { Prothrombin } \\
\text { time, s }\end{array}$} & 11.3 & 11 & $12^{\ddagger}$ & 11 & 10.3 & $12.95^{\ddagger}$ & 11.20 & 11.10 \\
\hline & $\begin{array}{l}(10.6- \\
12.2)\end{array}$ & $(10.28-11.8)$ & $(11-12.93)$ & $(10-13.2)$ & $(9.4-11.4)$ & $(11-15.9)$ & $\begin{array}{l}(10.70- \\
11.80)\end{array}$ & $(10.70-11.8)$ \\
\hline$\geq 16$ & 11(1.7) & $4(1.1)$ & $7(2.9)$ & 19(5.6) & $7(2.0)$ & $12(5.4)^{\dagger}$ & $4(1.2)$ & $2(0.8)$ \\
\hline \multirow{2}{*}{$\begin{array}{l}\text { D-dimer, } \\
\mu \mathrm{g} / \mathrm{mL}\end{array}$} & 0.9 & 0.64 & $1.72^{\ddagger}$ & 2.03 & 1.19 & $3.39 \neq$ & 0.32 & 0.32 \\
\hline & $\begin{array}{l}(0.47- \\
2.14)\end{array}$ & $(0.4-1.27)$ & $(0.79-8)$ & $\begin{array}{l}(0.65- \\
7.06)\end{array}$ & $(0.5-3.84)$ & $(1.6-27.5)$ & $\begin{array}{l}(0.17- \\
0.70)\end{array}$ & $(0.19-0.63)$ \\
\hline$>0.5$ & $470(72.1)$ & $255(63.1)$ & $215(86.5)^{\ddagger}$ & $465(138.0)$ & $265(74.4)$ & $200(89.3)^{\ddagger}$ & 108(32.0) & $78(30.5)$ \\
\hline \multirow[t]{2}{*}{$\begin{array}{l}\text { Serum } \\
\text { ferritin, } \mu \mathrm{g} / \mathrm{L}\end{array}$} & 628 & 493 & $921^{\ddagger}$ & 681 & 446 & $1204^{\ddagger}$ & 585 & 487 \\
\hline & $\begin{array}{l}(359- \\
1266)\end{array}$ & $(283-896)$ & $(511-1858)$ & $\begin{array}{l}(333- \\
1805)\end{array}$ & $(243-982)$ & $(550-2000)$ & $\begin{array}{l}(308- \\
1039)\end{array}$ & $(262-900)$ \\
\hline$>300$ & $521(79.9)$ & 292(72.2) & $229(92.4)^{\ddagger}$ & $458(135.9)$ & $247(69.4)$ & $211(94.2)^{\ddagger}$ & $256(76.0)$ & 178(69.5) \\
\hline \multirow[t]{2}{*}{ IL-6, pg/mL } & 7.52 & 7.33 & $8.43^{\ddagger}$ & 7.96 & 7.51 & $9.28^{\ddagger}$ & 7.32 & 7.22 \\
\hline & $\begin{array}{l}(5.85- \\
10.15)\end{array}$ & $(5.69-9.71)$ & $(6.12-12.04)$ & $\begin{array}{l}(6.18- \\
11.09)\end{array}$ & $(5.78-8.9)$ & $(6.73-18.58)$ & $\begin{array}{l}(5.67- \\
10.09)\end{array}$ & $(5.52-9.75)$ \\
\hline$>7$ & $377(57.8)$ & $218(54)$ & $159(64.3)^{\dagger}$ & $366(108.6)$ & $206(57.9)$ & $160(71.4)^{\dagger}$ & $180(53.4)$ & $133(52.0)$ \\
\hline \multirow[t]{2}{*}{$\mathrm{PCT}^{\mathrm{d}}, \mathrm{ng} / \mathrm{mL}$} & 0.05 & 0.05 & $0.08^{\ddagger}$ & 0.05 & 0.05 & $0.41^{\ddagger}$ & 0.05 & 0.05 \\
\hline & $\begin{array}{l}(0.05- \\
0.09)\end{array}$ & $(0.05-0.05)$ & $(0.05-0.19)$ & $\begin{array}{l}(0.05- \\
0.66)\end{array}$ & $(0.05-0.06)$ & $(0.05-1.78)$ & $\begin{array}{l}(0.05- \\
0.10)\end{array}$ & $(0.05-0.07)$ \\
\hline$>0.5$ & $29(4.4)$ & $9(2.2)$ & $20(7.9)^{\ddagger}$ & $141(41.8)$ & $43(12.1)$ & $98(43.8)^{\ddagger}$ & 13(3.9) & $2(0.8)$ \\
\hline \multirow[t]{2}{*}{ CRP, mg/L } & 42 & 23.5 & $78.6^{\ddagger}$ & 6.8 & 3.5 & $34.5^{\ddagger}$ & 11.60 & 9.99 \\
\hline & $\begin{array}{l}(12.15- \\
100.5)\end{array}$ & $(5.7-63)$ & $(39.43-148.4)$ & $\begin{array}{l}(1.58- \\
75.68)\end{array}$ & $(1-21.3)$ & $(4.5-160)$ & $\begin{array}{l}(5.01- \\
33.05)\end{array}$ & $(4.12-23.97)$ \\
\hline$>5$ & $551(84.5)$ & $313(77.5)$ & $238(96)^{\ddagger}$ & $319(94.7)$ & $156(43.8)$ & $163(72.8)^{\ddagger}$ & $254(75.4)$ & 181(70.7) \\
\hline \multirow[t]{2}{*}{$\mathrm{SAA}^{\mathrm{e}}, \mathrm{mg} / \mathrm{L}$} & 186.1 & 171.2 & $195.2^{\ddagger}$ & 45.3 & 20.4 & $114.8^{\ddagger}$ & - & - \\
\hline & $\begin{array}{l}(86.05- \\
249.8)\end{array}$ & $(58.2-254.4)$ & $(154-241.58)$ & $(6.6-207.8)$ & $(3.7-182.2)$ & $(14.7-223.3)$ & & \\
\hline$>10$ & $561(91.5)$ & $323(87.1)$ & $238(98.3)^{\ddagger}$ & 401(119.0) & $217(61.0)$ & $184(82.1)^{\ddagger}$ & & \\
\hline
\end{tabular}

Data are median $\left(\mathrm{P}_{25}-\mathrm{P}_{75}\right)$, mean $\pm \mathrm{SD}, \mathrm{n}(\%)$, or $\mathrm{n} / \mathrm{N}(\%) . P$ values were calculated by Mann-Whitney $\mathrm{U}$ test, $\mathrm{t}$ test, $\chi^{2}$ test, or Fisher's exact test, as appropriate.

a NLR: Neutrophils to Lymphocytes Ratio; b PLR: Platelets to Lymphocytes Ratio; ${ }^{\mathrm{c}}$ LDH: Lactate dehydrogenase; ${ }^{\mathrm{d}}$ PCT: Procalcitonin; ${ }^{\mathrm{e}}$ SAA: Serum amyloid $/$ $+P<0.05,{ }^{\ddagger} P<0.01$. 


\begin{tabular}{|c|c|c|c|c|c|c|c|c|}
\hline \multirow[b]{2}{*}{ Albumin, g/L } & \multicolumn{6}{|c|}{ Discovery dataset } & \multicolumn{2}{|c|}{ Validation dataset } \\
\hline & 31.3 & 32.7 & $29.1^{\ddagger}$ & 33.2 & 34.1 & $31^{\ddagger}$ & 42.01 & 42.44 \\
\hline & $\begin{array}{l}(28.5- \\
34.7)\end{array}$ & $(30.1-36.2)$ & $(26.9-31.7)$ & $(30-36.9)$ & $(31.4-37.8)$ & $(27.5-34.3)$ & $\begin{array}{l}(38.70- \\
45.30)\end{array}$ & $(39.58-45.5)$ \\
\hline$<35$ & $491(76.4)$ & $266(67.7)$ & $225(90)^{\ddagger}$ & $371(110.1)$ & 197(55.3) & $174(77.7)^{\ddagger}$ & $22(6.5)$ & $10(3.9)$ \\
\hline \multirow[t]{2}{*}{$\begin{array}{l}\text { CD4 + T cell, / } \\
\mu \mathrm{L}\end{array}$} & 342 & 390 & $217^{\ddagger}$ & - & - & - & - & - \\
\hline & $\begin{array}{l}(187- \\
566)\end{array}$ & $(286-594)$ & $(144.3-380.8)$ & & & & & \\
\hline \multirow[t]{2}{*}{$\begin{array}{l}\text { CD8 + T cell, / } \\
\mu \mathrm{L}\end{array}$} & 205 & 248 & $122.5^{\ddagger}$ & - & - & - & - & - \\
\hline & $\begin{array}{l}(113- \\
314)\end{array}$ & $(142-373)$ & $(58-178.8)$ & & & & & \\
\hline
\end{tabular}

Data are median $\left(\mathrm{P}_{25}-\mathrm{P}_{75}\right)$, mean $\pm \mathrm{SD}, \mathrm{n}(\%)$, or $\mathrm{n} / \mathrm{N}(\%)$. $P$ values were calculated by Mann-Whitney $\mathrm{U}$ test, $\mathrm{t}$ test, $\chi^{2}$ test, or Fisher's exact test, as appropriate.

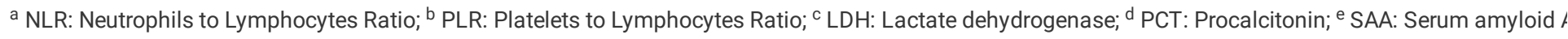

$+P<0.05,{ }^{\ddagger} P<0.01$.

In the validation stage (data was lacking at end hospitalization), admission levels of leukocytes, neutrophils, basophils, NLR, PLR, LDH, high-sensitivity cardiac troponin I, serum ferritin, IL-6, PCT and CRP were clearly elevated in severe-to- critically ill cases compared with mild-to-moderate patients; comparing to mildto-moderate group, lymphocytes, eosinophils and albumin were greatly reduced in severe-to-critical groups (Fig. 2a-q and Table 2).

\section{Correlation networks analysis of the published variables with the severity of COVID-19}

The correlation of all selected indexes were analyzed with Cytoscape software (https://cytoscape.org/). The positive indexes were shown with a red background, the negative ones were shown with a blue background, and the line with thick or fine represented the correlation of strong or weak, respectively. On hospital admission, strong positive correlations between NLR and the severity of COVID-19 were identified, followed by LDH; meanwhile, we observed a strong negative correlation between $\mathrm{SaO}_{2}$ and disease severity both in the discovery and validation stages (Fig. 3).

\section{Principal component analysis of variables for explaining proportion to the severity of COVID-19}

A biplot via PCA showed the configuration of indexes on hospital admission and at end hospitalization in Fig. 4. It is possible to condense several correlated variables into two composite parameters by this method which facilitates prediction of dichotomous endpoints. In this study, we first revealed that PCA is a well-established analytical method for severity stratification of COVID-19 patients. Plots of individual component scores for the first principal component (PC1) versus the second principal component (PC2) were provided. On hospital admission (Fig. 4A), PC1 and PC2 showed more marked separated severe-tocritical cases from mild-to-moderate patients. Combinations of PC1 and PC2 could explain 43.4\% proportion of the whole variances. Twelve predictors significantly associated positively or negatively with PC1, having the biggest positive and negative contribution, respectively. Notably, the spectrum of indexes in the PCA was consistent with what was shown in the correlation networks in the discovery stage (Fig. 3). At end hospitalization, mild-to-moderate patients and severe-to-critical cases present modest separated association (Fig. 4B). However, in the validation stage, PC1 and PC2 showed a weak separation of relations between the two groups (Fig. 4C).

\section{Independent Predictors And Roc Analysis On Hospital Admission}

The independent predictors were identified from previous studies ${ }^{5-19}$. We reanalyzed whether these published predictors could be validated in the present study. As indicated in Fig. 5, NLR, PLR, lymphocyte, CRP, IL-6, LDH, serum ferritin, CD4 + T cell, CD8 + T cell, high-sensitivity cardiac troponin I, albumin and Ddimer were found to be independent predictors for COVID-19 severity. ROC analysis of single independent variable was calculated using the expression levels at hospital admission (uni-AuROC). Results showed that the AuROC was 0.782 for NLR, follow by LDH of 0.765 and CD8 + T cell of 0.753 . The AuROC of other indexes varied from 0.585 to 0.730 in the discovery stage (Fig. 5a-I and Fig. 8a). With a cut-off value of 7.0, NLR exhibited sensitivity $72.3 \%$, specificity $74.4 \%$, correct classification ratio (CCR) $73.6 \%$, positive prediction value (PPV) $65.3 \%$, and negative prediction value (NPV) $80.2 \%$ in the discovery stage (Table 3 ), and NLR still demonstrated to be the biggest AuROC of 0.794 in the validation stage (Fig. 5a-j and Fig. 8a). Then, we tested the different combination of NLR and the other one risk parameter for the prediction of disease (bi-AuROC) (Fig. 6). In the discovery study, combination of $\mathrm{NLR}$ and $\mathrm{SaO} \mathrm{O}_{2}$ showed the highest AuROC of 0.901 , followed by the combination of NLR and LDH (0.807), then NLR and D-dimer (0.800) (Fig. 6a-o and Fig. 8b). NLR + SaO $2, \mathrm{NLR}+\mathrm{complication}$ and $\mathrm{NLR}+$ age were the top three highest AuROC of $0.876,0.830$ and 0.819 in the validation stage, respectively (Fig. $6 \mathrm{a}-\mathrm{I}$ and Fig. $8 \mathrm{~b}$ ). With a cut-off value of 0.532 , $\mathrm{NLR}+\mathrm{SaO}_{2}$ exhibited $84.2 \%$ sensitivity, $88.4 \%$ specificity, $86.8 \%$ correct classification ratio (CCR), $81.8 \%$ positive prediction value (PPV), and $90.2 \%$ negative prediction value (NPV) in the discovery stage (Table 4). 
Table 3

Performance of cut-off scores in discovery dataset (NLR).

\begin{tabular}{|c|c|c|c|c|c|c|c|c|c|}
\hline Cut-off & Y predict & AUC & Sensitivity & Specificity & CCR & PPV & NPV & LR+ & LR- \\
\hline & & & (\%) & (\%) & (\%) & (\%) & (\%) & & \\
\hline$>=6.0$ & $>=0.302$ & 0.712 & 75.9 & 66.5 & 70.3 & 60.1 & 80.6 & 2.27 & 0.36 \\
\hline$>=6.5$ & $>=0.317$ & 0.717 & 73.7 & 69.6 & 71.3 & 61.8 & 80.0 & 2.43 & 0.38 \\
\hline$>=6.8$ & $>=0.327$ & 0.723 & 72.3 & 72.3 & 72.3 & 63.4 & 79.7 & 2.61 & 0.38 \\
\hline$>=6.9$ & $>=0.330$ & 0.726 & 72.3 & 73.0 & 72.7 & 64.0 & 79.8 & 2.67 & 0.38 \\
\hline$>=7.0 *$ & $>=0.333^{*}$ & 0.734 & 72.3 & 74.4 & 73.6 & 65.3 & 80.2 & 2.82 & 0.37 \\
\hline$>=7.1$ & $>=0.336$ & 0.727 & 70.5 & 74.9 & 73.1 & 65.1 & 79.2 & 2.81 & 0.39 \\
\hline$>=7.2$ & $>=0.340$ & 0.725 & 70.1 & 74.9 & 73.0 & 65.0 & 79.0 & 2.79 & 0.40 \\
\hline$>=7.5$ & $>=0.350$ & 0.728 & 69.1 & 76.6 & 73.6 & 66.2 & 78.8 & 2.95 & 0.40 \\
\hline$>=8.0$ & $>=0.366$ & 0.729 & 67.3 & 78.5 & 74.0 & 67.5 & 78.3 & 3.12 & 0.42 \\
\hline$>=8.5$ & $>=0.383$ & 0.726 & 65.5 & 79.7 & 74.0 & 68.2 & 77.6 & 3.22 & 0.43 \\
\hline$>=9.0$ & $>=0.400$ & 0.719 & 63.0 & 80.9 & 73.7 & 68.6 & 76.6 & 3.29 & 0.46 \\
\hline$>=10.0$ & $>=0.436$ & 0.700 & 57.6 & 82.5 & 72.6 & 68.7 & 74.5 & 3.30 & 0.51 \\
\hline
\end{tabular}


Table 4

Performance of cut-off scores in discovery dataset (NLR + 02).

\begin{tabular}{|c|c|c|c|c|c|c|c|c|c|}
\hline$Y$ & $Y$ & AUC & Sensitivity & Specificity & CCR & PPV & NPV & LR+ & LR- \\
\hline cut-off & predict & & (\%) & (\%) & (\%) & (\%) & (\%) & & \\
\hline$>=-0.847$ & $>=0.300$ & 0.844 & 87.1 & 81.6 & 83.7 & 74.1 & 91.3 & 4.74 & 0.16 \\
\hline$>=-0.800$ & $>=0.310$ & 0.851 & 87.1 & 83.1 & 84.6 & 75.7 & 91.4 & 5.16 & 0.16 \\
\hline$>=-0.754$ & $>=0.320$ & 0.857 & 87.1 & 84.4 & 85.4 & 77.1 & 91.5 & 5.58 & 0.15 \\
\hline$>=-0.731$ & $>=0.325$ & 0.856 & 86.3 & 84.9 & 85.4 & 77.5 & 91.1 & 5.71 & 0.16 \\
\hline$>=-0.708$ & $>=0.330$ & 0.859 & 85.8 & 85.9 & 85.9 & 78.6 & 90.9 & 6.08 & 0.16 \\
\hline$>=-0.686$ & $>=0.335$ & 0.860 & 85.0 & 86.9 & 86.2 & 79.7 & 90.6 & 6.49 & 0.17 \\
\hline$>=-0.663$ & $>=0.340$ & 0.861 & 85.0 & 87.2 & 86.3 & 80.0 & 90.6 & 6.62 & 0.17 \\
\hline$>=-0.619$ & $>=0.350$ & 0.857 & 84.2 & 87.2 & 86.0 & 79.8 & 90.1 & 7.39 & 0.17 \\
\hline$>=-0.575$ & $>=0.360$ & 0.860 & 84.2 & 87.9 & 86.5 & 80.8 & 90.2 & 6.96 & 0.18 \\
\hline$>=-0.532$ & $>=0.370 *$ & 0.863 & 84.2 & 88.4 & 86.8 & 81.5 & 90.2 & 7.27 & 0.18 \\
\hline$>=-0.490$ & $>=0.380$ & 0.860 & 82.9 & 89.2 & 86.8 & 82.2 & 89.6 & 7.66 & 0.19 \\
\hline$>=-0.447$ & $>=0.390$ & 0.862 & 82.9 & 89.4 & 87.0 & 82.6 & 89.7 & 7.84 & 0.19 \\
\hline$>=-0.405$ & $>=0.400$ & 0.863 & 82.9 & 89.7 & 87.1 & 82.9 & 89.7 & 8.03 & 0.19 \\
\hline$>=-0.364$ & $>=0.410$ & 0.855 & 81.3 & 89.7 & 86.5 & 82.6 & 88.8 & 7.87 & 0.21 \\
\hline$>=-0.323$ & $>=0.420$ & 0.846 & 79.6 & 89.7 & 86.9 & 82.3 & 87.9 & 7.71 & 0.23 \\
\hline$>=-0.282$ & $>=0.430$ & 0.845 & 78.7 & 90.2 & 85.9 & 82.9 & 87.5 & 8.02 & 0.23 \\
\hline \multicolumn{10}{|c|}{ * Yielded the largest AUC and a balance between sensitivity and specificity at this cut-off score. } \\
\hline \multicolumn{10}{|c|}{$\begin{array}{l}\mathrm{AUC}=\text { area under the receiver operating characteristic curve; } C \mathrm{CR}=\text { Correct Classification ratio; } \mathrm{PPV}=\text { positive predictive value; NPV = negative predictive } \\
\text { value; } \mathrm{LR}+=\text { positive likelihood ratio; } \mathrm{LR}-=\text { negative likelihood ratio. }\end{array}$} \\
\hline \multicolumn{10}{|c|}{$Y_{\text {cut-off }}=0.0941957 * N L R-0.4117971 * \mathrm{SaO}_{2}+37.3027$} \\
\hline
\end{tabular}

\section{AuROC comparisons of different models in prediction of severe COVID-19}

To compare the accuracy of different published models with ours in severity of COVID-19 prediction ( $N L R+\mathrm{SaO}_{2}$ as the reference model), different ROC curve analysis was performed and the difference in AuROCs was tested by using our data. The AuROCs of NLR $+\mathrm{SaO}, \mathrm{PCA}, \mathrm{Zhu}$ et al. model, Jiang et al. model, Bi et al. model and Henry et al. model were 0.901 (95\% Cl: $0.874-0.928), 0.865(95 \% \mathrm{Cl}: 0.830-0.899)(p=0.09), 0.739(95 \% \mathrm{Cl}: 0.697-0.781)(p=0.002), 0.755$ (95\% Cl: $0.677-0.834)(p=0.003), 0.749$ (95\% Cl: 0.708-0.790) ( $p=0.009)$ and 0.817 (95\% Cl: $0.780-0.855)(p=0.02)$, respectively. It showed that the AuROC of $\mathrm{NLR}+\mathrm{SaO}_{2}$ to predict $\mathrm{COVID}-19$ severity was significantly higher than these published models, which demonstrated the excellent predictive power for the severity of disease, and there was no significantly difference between NLR $+\mathrm{SaO}_{2}$ and PCA in the discovery stage (Table 5 discovery dataset, Fig. $7 a-f$ and Fig. 8c). The nearly consistent direction of effects were revealed in the validation stage (except Henry et al. model showed no significant difference compared with $\left.\mathrm{NLR}+\mathrm{SaO}_{2}\right)($ Table 5 validation dataset, Fig. $7 \mathrm{~g}-\mathrm{k}$ and Fig. $8 \mathrm{c})$. 
Table 5

AuROC comparisons of different predictive models

\begin{tabular}{|c|c|c|c|c|c|c|}
\hline Model & AuROC & $95 \% \mathrm{Cl}$ & P value & Sensitivity & Specificity & C-index ${ }^{a}$ \\
\hline & & & & $(\%)$ & $(\%)$ & \\
\hline \multicolumn{7}{|c|}{ Discovery dataset } \\
\hline $\mathrm{NLR}+\mathrm{SaO}_{2}$ & 0.901 & $(0.874-0.928)$ & Reference & 84.2 & 88.4 & 0.868 \\
\hline $\mathrm{PCA}^{\mathrm{b}}$ & 0.865 & $(0.830-0.899)$ & 0.09 & 75.9 & 83.7 & 0.803 \\
\hline Zhu et al. ${ }^{c}$ & 0.739 & $(0.697-0.781)$ & 0.002 & 66.2 & 70.2 & 0.685 \\
\hline Jiang et al. ${ }^{d}$ & 0.755 & $(0.677-0.834)$ & 0.003 & 70.0 & 72.1 & 0.714 \\
\hline Bi et al. e & 0.749 & $(0.708-0.790)$ & 0.009 & 67.0 & 70.8 & 0.693 \\
\hline Henry et al. ${ }^{f}$ & 0.817 & $(0.780-0.855)$ & 0.02 & 72.4 & 78.4 & 0.759 \\
\hline \multicolumn{7}{|c|}{ Validation dataset } \\
\hline $\mathrm{NLR}+\mathrm{SaO}_{2}$ & 0.876 & $(0.826-0.926)$ & Reference & 79.1 & 85.2 & 0.837 \\
\hline PCA ${ }^{b}$ & 0.858 & $(0.792-0.924)$ & 0.92 & 70.0 & 81.2 & 0.783 \\
\hline Zhu et al. ${ }^{c}$ & 0.743 & $(0.667-0.818)$ & 0.027 & 68.3 & 70.8 & 0.702 \\
\hline Bi et al. e & 0.641 & $(0.561-0.722)$ & 0.0002 & 54.1 & 65.3 & 0.627 \\
\hline Henry et al. ${ }^{f}$ & 0.829 & $(0.775-0.884)$ & 0.17 & 70.8 & 82.9 & 0.795 \\
\hline \multicolumn{7}{|c|}{ a C-index = correctly diagnosed / total effective number; } \\
\hline \multicolumn{7}{|c|}{ b PCA: assessing the severity of COVID-19 established upon principal component analysis (PCA); } \\
\hline \multicolumn{7}{|c|}{ c Zhu et al. reported that assessing the severity of COVID-19 established upon IL-6, CRP and hypertension; } \\
\hline \multicolumn{7}{|c|}{ d Jiang et al. reported that assessing the severity of COVID-19 established upon the counts of CD8 + T and CD4+T cells; } \\
\hline \multicolumn{7}{|c|}{ e $\mathrm{Bi}$ et al. reported that assessing the severity of COVID-19 established upon initial fibrinogen to albumin ratio (FAR) and platelet Count; } \\
\hline
\end{tabular}

\section{Discussion}

In the present study, NLR was verified to be the most independent outcome predictor in patients with COVID-19. For NLR had the biggest AuROC calculations with higher sensitivity, specificity, and CCR compared with the others significant published variables in both discovery and validation datasets. Usually a single index does not adequately predict disease severity, but incorporating too many variables would increase the risk of overfitting the model when building prediction models as the review on BMJ described. We therefore further performed a correlation analysis on NLR and the others published indexes. The findings of this study suggested that the combination of NLR and $\mathrm{SaO}_{2}$ performed better predictability value than other two indexes in the severity of COVID19 patients. In addition, PCA was identified to be an effective tool to predict the severity of COVID-19 cases. To evaluate the effectiveness of NLR + SaO ${ }_{2}$ and PCA, we compared them with other predictive models in the same dataset, and validated them on an independent dataset. It concluded that NLR $+\mathrm{SaO} \mathrm{O}_{2}$ is an appropriate and promising method for predicting COVID-19 severity, followed by PCA. Application of these models might be beneficial to delay or halt the progression of the disease.

The comparison of PCA and $\mathrm{NLR}+\mathrm{SaO}_{2}$ : PCA incorporates more comprehensive variables and shows better model stability, while being able to good for solving the overfitting, interaction and co-collinearity problems of the independent variables as the review published on BMJ mentioned. It is a data reduction technique that is better used for multivariate dimensionality reduction; however, PCA is unsuitable for clinical application for too many variables involved. NLR $+\mathrm{SaO}_{2}$ are the simplicity of the variables combination, the absence of variables interaction and co-collinearity, and the most importantly, the clinical application is easy and quick and of great value.

This retrospective study identified several risk factors for COVID-19 patients. In particular, elevated levels of leukocytes, neutrophils, NLR, CRP, IL-6, lactate dehydrogenase, D-dimer and lymphocytopenia etc were more commonly seen in severe-to-critical COVID-19 illness. We found that these above risk factors were associated with the outcomes of COVID-19 and were consistent with studies published ${ }^{18,22}$. The pathological mechanism of COVID-19 has not been fully uncovered. Our findings were consistent with those of numerous studies on the relationship between elevated NLR and virus infection ${ }^{18,23}$. Elevated neutrophil count was observed in patients with severe illness compared to those with non-severe illness ${ }^{24}$. Raised neutrophil count might result from secondary virus-related inflammatory factors, excessive inflammatory stress or glucocorticoids use and contribute to exacerbate disease progression in 
patients with COVID-19. Moreover, reduction in peripheral lymphocyte count was also commonly observed in COVID-19 cases, which was considered a possible critical factor associated with disease severity and mortality ${ }^{25,26}$. Lymphocyte plays pivotal roles in human immune response caused by viral infection, whereas systematic inflammation significantly depresses cellular immunity. In our study, lymphocytopenia was identified in severe COVID-19 cases. The possible reasons is that lymphocytes are depleted, as the virus is engulfed, or that lymphocytes could be directly infected and destroyed by SARS-CoV-2, because the coronavirus angiotensin-converting enzyme 2 (ACE2) receptor was widely available in lymphocytes ${ }^{27}$. A low oxygen saturation ( $(\mathrm{SaO})_{2}$ ) is one of the main criteria for the definition of a severe case. $\mathrm{SaO}_{2}$ was found as another candidate marker of progressive severity ${ }^{28}$ and also validated in the present study. We comprehensively investigated the relationship between risk factors and the severity of COVID-19 and first concluded that the integration of NLR with $\mathrm{SaO}_{2}$ may lead to improved prediction. The applicable thresholds for $\mathrm{NLR}+\mathrm{SaO}_{2}$ were observed using the AuROC. The optimal threshold at 0.532 for $\mathrm{NLR}+$ $\mathrm{SaO}_{2}$ showed superior predictive possibilities to separate severe-to-critical cases from mild-to-moderate patients, which had the highest of sensitivity and specificity and the largest of AuROC. For instance, when NLR $=7$ and $\mathrm{SaO}_{2}=92,38 \%$ of the COVID-19 patients were predicted as severe-to-critical patients. So, these patients must be closely attended by clinicians.

To gain a comprehensive view of the selected parameters, a correlation network analysis was conducted, and identified that a modest to high correlations were existed between variables. It provided clues for the limitation of statistical methods used for prediction models in a traditional way. PCA is multivariate techniques to examine the internal structure of complicated datasets and to explore the interrelations among variables. It is used to emphasize variation and reveal strong patterns in a large dataset and allows for the identification of uncorrelated eigenvectors to describe large multidimensional datasets. PCA may be valuable in studies of COVID-19 and we first identified that PCA is an effective tool with which to predict the severity of COVID-19. Application of this model might be beneficial to separate severe-to-critical cases from mild-to-moderate patients. The standardized procedure of PCA in the evaluation of the severity of COVID-19 included: (1) compute the composite scores for the combined effects that accurately capture pathological expression; (2) bring out which variables have the most variation in COVID-19.

Subsequent AuROC comparisons analysis were conducted among diferent predictive models, which included $\mathrm{NLR}+\mathrm{SaO}, \mathrm{PCA}$ and published models. It showed that $\mathrm{NLR}+\mathrm{SaO}_{2}$ and PCA could better distinguish mild-to-moderate cases and severe-to-critical patients.

Our study has some limitations. First, because this study was retrospective, other markers such as IL-1Ra, IL-10, IFN- - -induced protein 10 (IFN- $\gamma-10$ ), monocyte chemotactic protein-3 (MCP-10), were not included in the study due to data unavailability. Therefore, their influences on outcomes might be underestimated. Second, severe-to-critical cases took a large percentage in the discovery stage, but mild-to-moderate patients played a dominant role in the validation stage, which might bring about selective bias. Finally, large-scale multicenter clinical researches are needed to be further investigated.

\section{Conclusions}

The present study showed that $\mathrm{NLR}+\mathrm{SaO}_{2}$ is an appropriate and promising method for predicting COVID-19 severity, followed by PCA. We compared them with other predictive models in the same dataset, and validated them on an independent dataset.

\section{Abbreviations}

PCA, Principal component analysis;

AuROC, The area under the curve of the receiver operating characteristics;

AUC, The area under the receiver operating characteristic curve;

ROC, Receiver operating characteristic;

NLR, Neutrophils to Lymphocytes Ratio;

PLR, Platelets to Lymphocytes Ratio;

LDH, Lactate dehydrogenase;

PCT, Procalcitonin;

SAA, Serum amyloid A;

CCR, correct classification ratio;

PPV, positive predictive value;

NPV, negative predictive value;

LR+, positive likelihood ratio;

LR-, negative likelihood ratio;

IQR, Interquartile range; 
CRP, C-reactive protein.

FAR , Fibrinogen to albumin ratio;

WBC, white blood cell / leukocyte;

CD4, CD4+T cells;

CD8, CD8+T cells.

\section{Declarations}

\section{Author contributions}

Wenju Lu, Nanshan Zhong, Qinghui Huang, and Qiaoxin Huang conceived and designed the article. E Guo, Minglong Fu, Xuejiao Yang, Zhuowei Li, Jingyi Xu, Yun Bai, Qiaoxin Huang, Xiaodan Zhang, Fei Liu, Liang Yuan, Xiaohui Xie, Qiongqiong Li, Bingxian Deng, Lingzhu Chen, Yongxuan Gao, Lan Wang, Zhou Cai, Zhanbei Zhu, Fanjie, Lin, Wei Liu and Hua Guo collected the clinical data. Zili Zhang and Xiaodan Zhang conducted the data and analyzed the clinical data. Zili Zhang, Xiansheng Zeng, Jian Wang wrote the manuscripts. Wenju Lu oversaw the completion of this study and edited the manuscript.

\section{Funding}

This study was supported by grants from the National Key R\&D Program of China (2016YFC0903700), National Natural Science Foundation of China (81520108001, 81770043 and 81700043), Guangzhou Department of Education (1201620007), Local Innovative and Research Teams Project of Guangdong Pearl River Talents Program (2017BT01S155), the Guangdong Natural Science Foundation (2020A1515010076), and the Research Projects of SKLRD (OP201808, QN-201706, QN-201917, OP-201912).

\section{Ethical Approval and Consent to participate}

All the procedures were approved by the ethics commission of the First Affiliated Hospital of Guangzhou Medical University (Institutional Review Board: 202092) and was performed in compliance with the Declaration of Helsinki. Verbal informed consent were obtained from all participants because the family members were in quarantine.

\section{Consent for publication}

Not applicable.

\section{Availability of Data and Materials:}

The datasets used during the current study are available from the corresponding author on reasonable request.

\section{Competing interests}

The authors have no conflict of interest to declare.

\section{Consent for publication}

Not applicable.

\section{Acknowledgements}

We sincerely thank all the healthcare providers fighting against this public crisis and all the patients involved in the study. We express sincere sympathies and deep condolences to the victims and bereaved families.

\section{References}

1. Epidemiology Working Group for Ncip Epidemic Response CCfDC, Prevention. [The epidemiological characteristics of an outbreak of 2019 novel coronavirus diseases (COVID-19) in China]. Zhonghua Liu Xing Bing Xue Za Zhi. 2020;41(2):145-151.

2. Yang X, Yu Y, Xu J, et al. Clinical course and outcomes of critically ill patients with SARS-CoV-2 pneumonia in Wuhan, China: a single-centered, retrospective, observational study. Lancet Respir Med. 2020;8(5):475-481.

3. Wynants L, Van Calster B, Collins GS, et al. Prediction models for diagnosis and prognosis of covid-19 infection: systematic review and critical appraisal. BMJ. 2020;369:m1328.

4. IT. J. Principal Component Analysis, 2nd ed. . Berlin Springer; 2002.

5. Diagnosis and Treatment Protocol for Novel Coronavirus Pneumonia (Trial Version 7). Chin Med J (Engl). 2020;133(9):1087-1095.

6. Yang Y, Shen C, Li J, et al. Plasma IP-10 and MCP-3 levels are highly associated with disease severity and predict the progression of COVID-19. J Allergy Clin Immunol. 2020;146(1):119-127 e114. 
7. Henry BM, de Oliveira MHS, Benoit S, Plebani M, Lippi G. Hematologic, biochemical and immune biomarker abnormalities associated with severe illness and mortality in coronavirus disease 2019 (COVID-19): a meta-analysis. Clin Chem Lab Med. 2020;58(7):1021-1028.

8. Liu F, Li L, Xu M, et al. Prognostic value of interleukin-6, C-reactive protein, and procalcitonin in patients with COVID-19. J Clin Virol. 2020;127:104370.

9. Zhu Z, Cai T, Fan L, et al. Clinical value of immune-inflammatory parameters to assess the severity of coronavirus disease 2019 . Int $\mathrm{J}$ Infect Dis. 2020;95:332-339.

10. Liu Z, Long W, Tu M, et al. Lymphocyte subset (CD4+, CD8+) counts reflect the severity of infection and predict the clinical outcomes in patients with COVID-19. J Infect. 2020.

11. Han H, Ma Q, Li C, et al. Profiling serum cytokines in COVID-19 patients reveals IL-6 and IL-10 are disease severity predictors. Emerg Microbes Infect. 2020;9(1):1123-1130.

12. Zhang JJY, Lee KS, Ang LW, Leo YS, Young BE. Risk Factors of Severe Disease and Efficacy of Treatment in Patients Infected with COVID-19: A Systematic Review, Meta-Analysis and Meta-Regression Analysis. Clin Infect Dis. 2020.

13. Zhao Y, Qin L, Zhang P, et al. Longitudinal COVID-19 profiling associates IL-1RA and IL-10 with disease severity and RANTES with mild disease. JCI Insight. 2020;5(13).

14. Tan C, Huang Y, Shi F, et al. C-reactive protein correlates with computed tomographic findings and predicts severe COVID-19 early. J Med Virol. 2020;92(7):856-862.

15. Jiang M, Guo Y, Luo Q, et al. T-Cell Subset Counts in Peripheral Blood Can Be Used as Discriminatory Biomarkers for Diagnosis and Severity Prediction of Coronavirus Disease 2019. J Infect Dis. 2020;222(2):198-202.

16. Bi X, Su Z, Yan H, et al. Prediction of severe illness due to COVID-19 based on an analysis of initial Fibrinogen to Albumin Ratio and Platelet count. Platelets. 2020;31(5):674-679.

17. Hou H, Zhang B, Huang $\mathrm{H}$, et al. Using IL-2R/lymphocytes for predicting the clinical progression of patients with COVID-19. Clin Exp Immunol. 2020;201(1):76-84.

18. Yang AP, Liu JP, Tao WQ, Li HM. The diagnostic and predictive role of NLR, d-NLR and PLR in COVID-19 patients. Int Immunopharmacol. $2020 ; 84: 106504$.

19. Zheng HY, Zhang M, Yang CX, et al. Elevated exhaustion levels and reduced functional diversity of T cells in peripheral blood may predict severe progression in COVID-19 patients. Cell Mol Immunol. 2020;17(5):541-543.

20. Luo X, Zhou W, Yan X, et al. Prognostic value of C-reactive protein in patients with COVID-19. Clin Infect Dis. 2020.

21. Krzanowski W. Principles of multivariate analysis. . New York: Oxford University; 2000.

22. Drent M, Cobben NA, Henderson RF, Wouters EF, van Dieijen-Visser M. Usefulness of lactate dehydrogenase and its isoenzymes as indicators of lung damage or inflammation. Eur Respir J. 1996;9(8):1736-1742.

23. Liu Y, Du X, Chen J, et al. Neutrophil-to-lymphocyte ratio as an independent risk factor for mortality in hospitalized patients with COVID-19. J Infect. 2020;81(1):e6-e12.

24. Barnes BJ, Adrover JM, Baxter-Stoltzfus A, et al. Targeting potential drivers of COVID-19: Neutrophil extracellular traps. J Exp Med. 2020;217(6).

25. Xu Z, Shi L, Wang Y, et al. Pathological findings of COVID-19 associated with acute respiratory distress syndrome. Lancet Respir Med. 2020;8(4):420-422.

26. Chan JF, Yuan S, Kok KH, et al. A familial cluster of pneumonia associated with the 2019 novel coronavirus indicating person-to-person transmission: a study of a family cluster. Lancet. 2020;395(10223):514-523.

27. Li MY, Li L, Zhang Y, Wang XS. Expression of the SARS-CoV-2 cell receptor gene ACE2 in a wide variety of human tissues. Infect Dis Poverty. 2020;9(1):45.

28. Assandri R, Buscarini E, Canetta C, Scartabellati A, Vigano G, Montanelli A. Laboratory Biomarkers Predicting COVID-19 Severity in the Emergency Room. Arch Med Res. 2020.

\section{Figures}



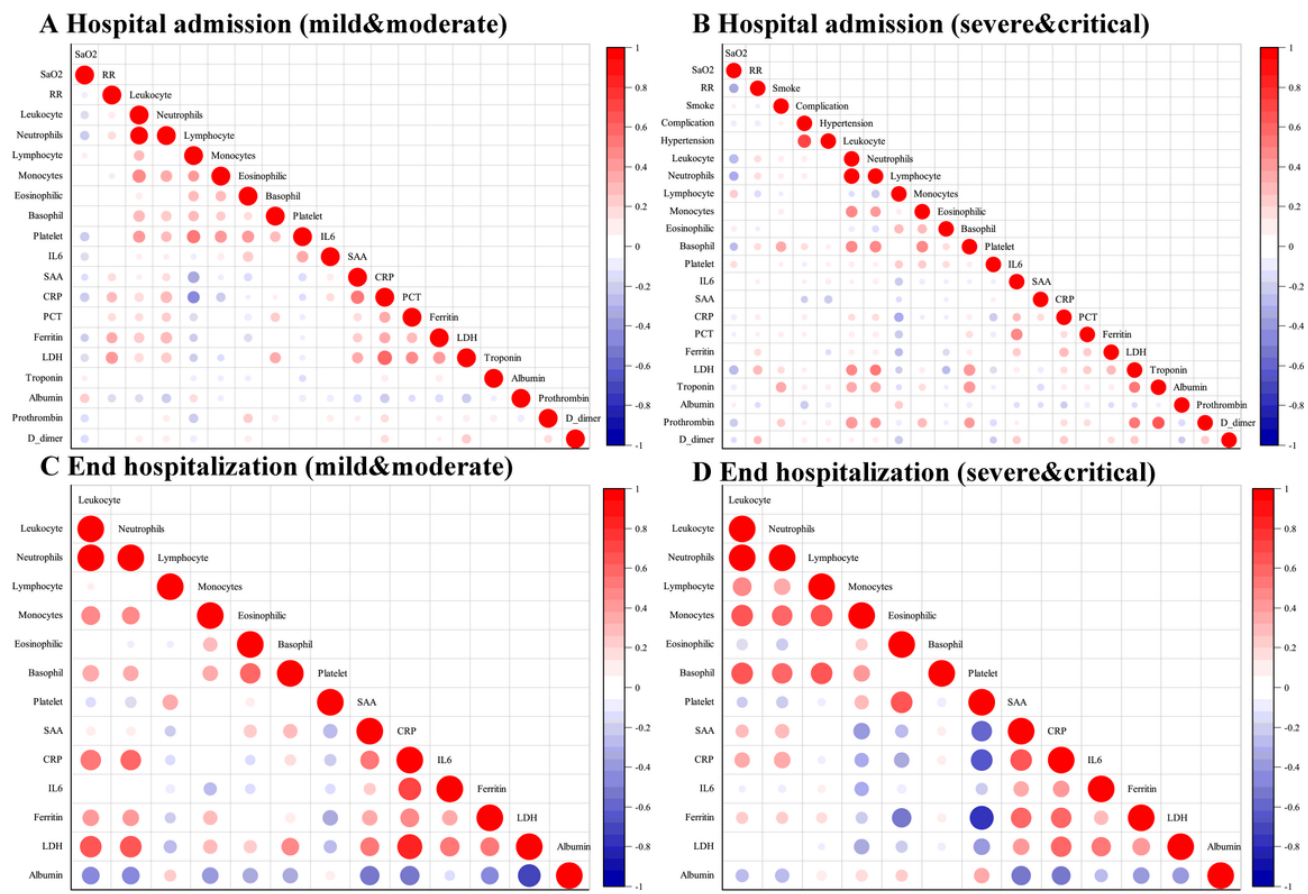

D End hospitalization (severe\&critical)
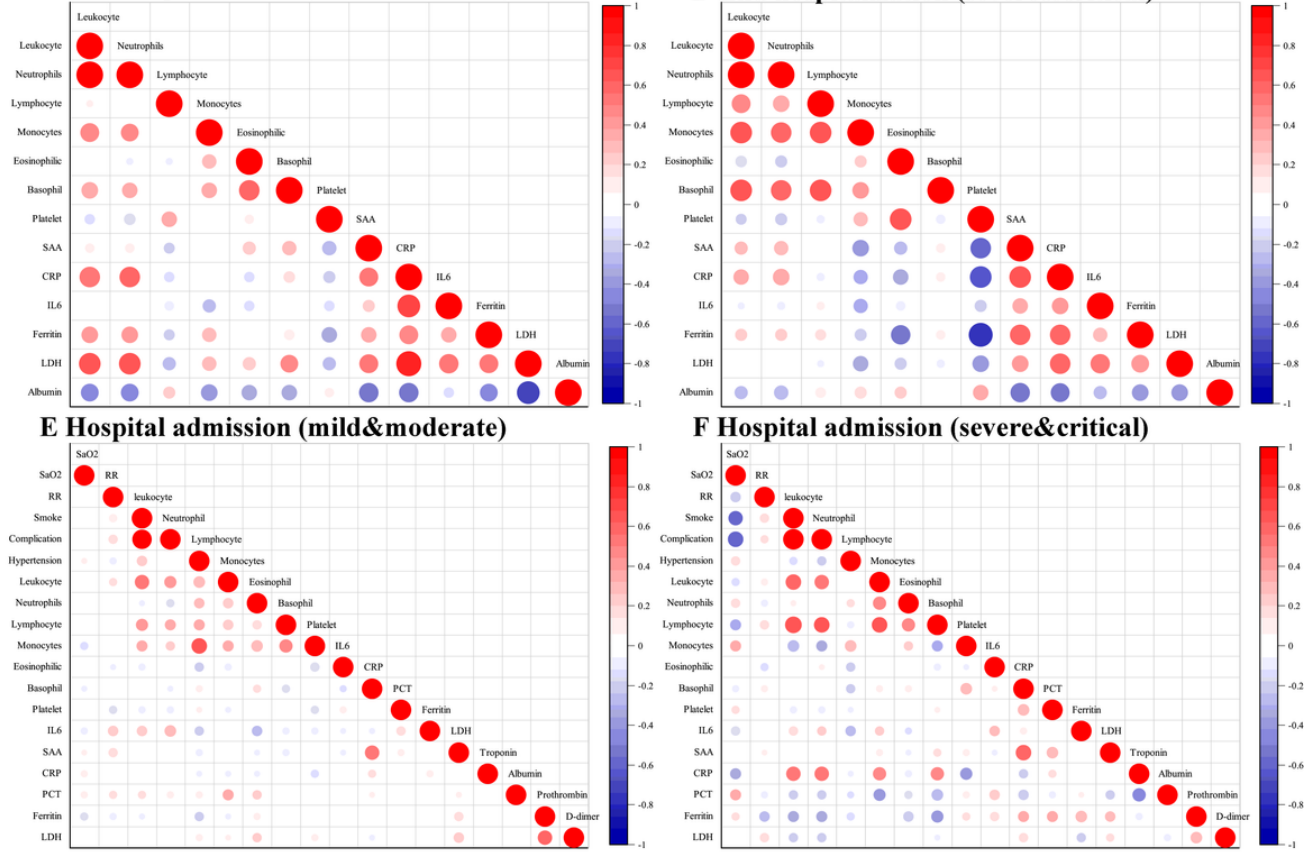

F Hospital admission (severe\&critical)

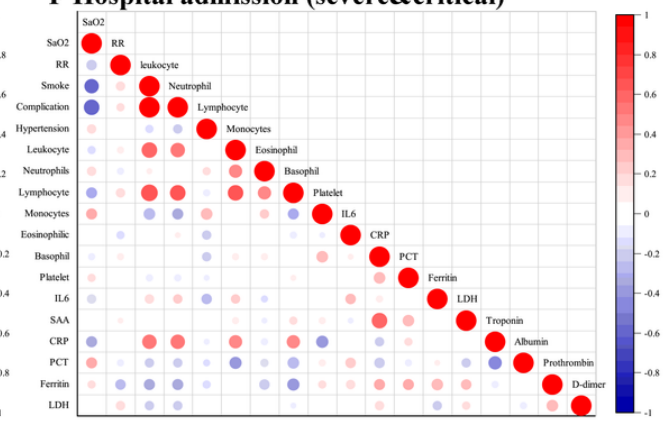

\section{Figure 1}

Heatmap of the correlation matrix. A) Correlation analysis of the published variables between each other in mild-to-moderate patients on hospital admission in the discovery stage; B) Correlation analysis of the published variables between each other in severe-to-critically ill patients on hospital admission in the discovery stage; C) Correlation analysis of the published variables between each other in mild-to-moderate cases at end hospitalization admission in the discovery stage; D) Correlation analysis of the published variables between each other in severe-to-critically ill patients at end hospitalization admission in the discovery stage; E) Correlation analysis of the published variables between each other in mild-to-moderate patients on hospital admission in the validation stage; F) Correlation analysis of the published variables between each other in severe-to-critically ill patients on hospital admission in the validation stage. Circle size and color indicated relevant strength, dark red and big circles denoted the positively and strong associations, light red and small circles denoted the positively and weak associations. 


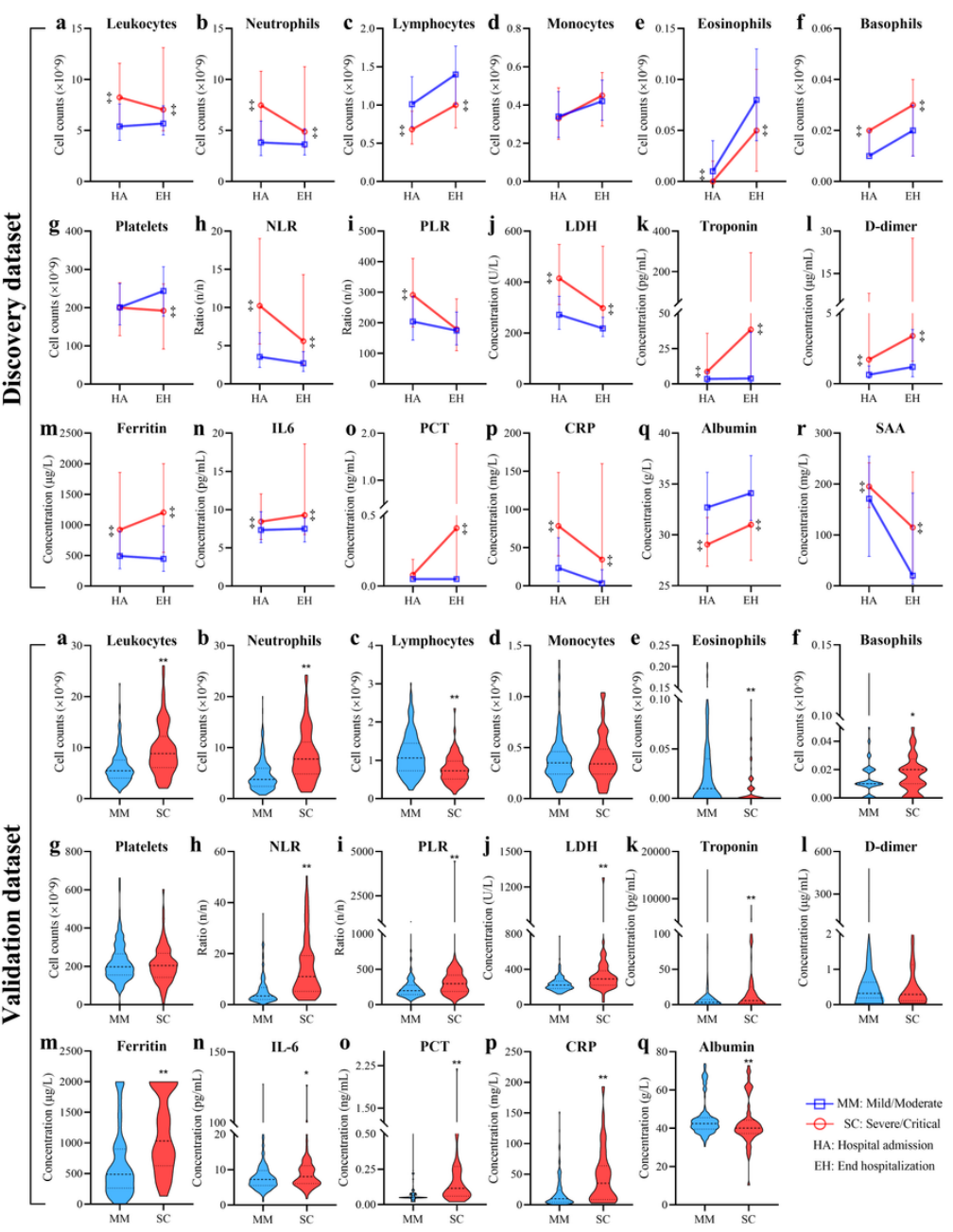

Figure 2

Dynamic changes or expression levels of of laboratory parameters in COVID-19 patients on hospital admission (HA) and end hospitalization (EH). Medium (P25-P75) value of the two time periods was showed in mild-to-moderate cases and severe-to-critically ill patients in the discovery stage (a-r). In the validation stage (data was lacking at end hospitalization), admission levels of variables were compared between the two groups (a-q). The significant difference between mild-to-moderate cases and severe-to-critically ill patients was compared using Mann-Whitney U test, $\chi^{2}$ test, or Fisher's exact test, as appropriate. $+p$ $<0.05, \neq \mathrm{p}<0.01$.

\section{A. Hospital admission}

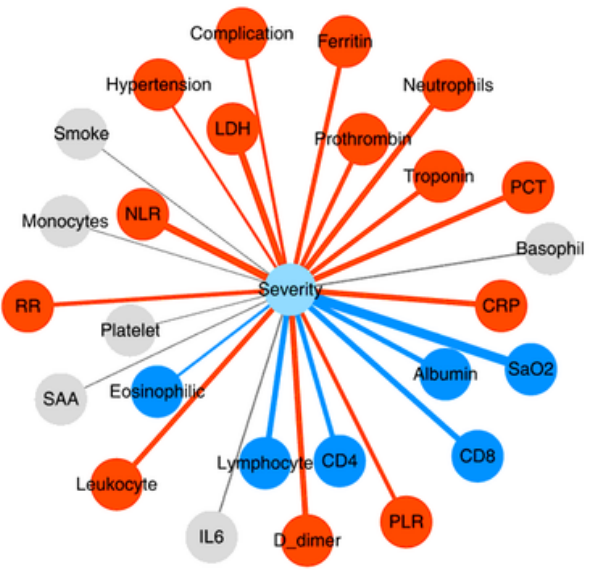

B. End hospitalization

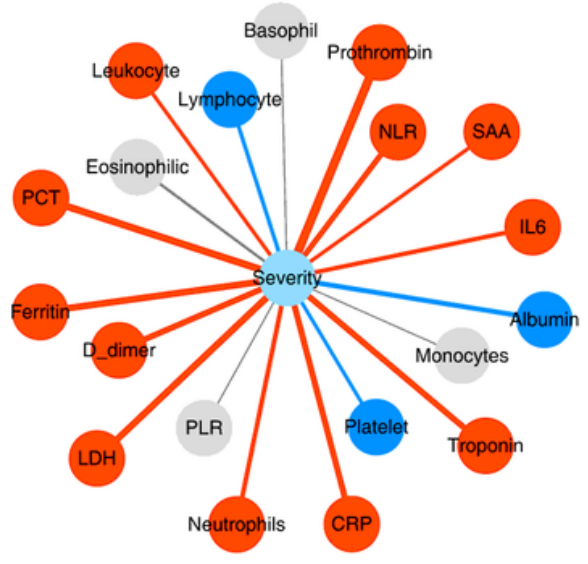

\section{Hospital admission}

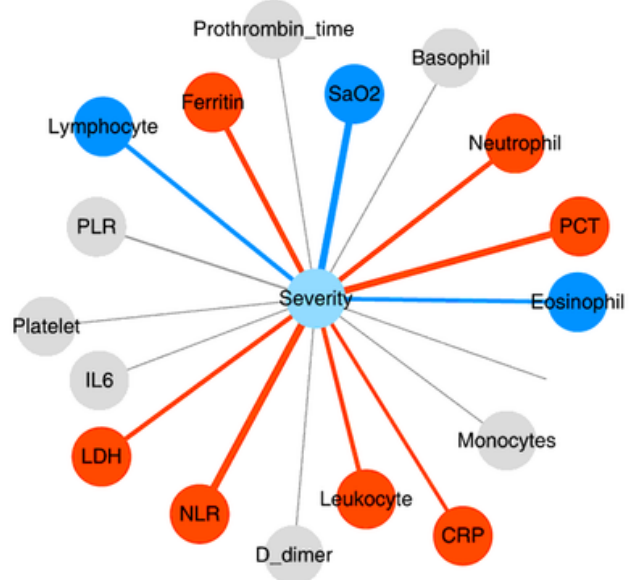

Figure 3 
Correlation networks analysis of the published variables with the severity of COVID-19. A) Correlation networks analysis of the published variables on hospital admission in the discovery stage; B) Correlation networks analysis of the published variables at end hospitalization in the discovery stage; C) Correlation networks analysis of the published variables on hospital admission in the validation stage. Nodes and line are colored based on positive or negative. Nodes represent different indexes. Thicker lines indicate more correlated indexes. Red lines and nodes represent significantly positive Spearman's correlation coefficients $\geq 0.30$; Blue lines and red nodes represent significantly negative Spearman's correlation coefficients $\leq-0.3$.

A. Bioplot (Hospital admission)

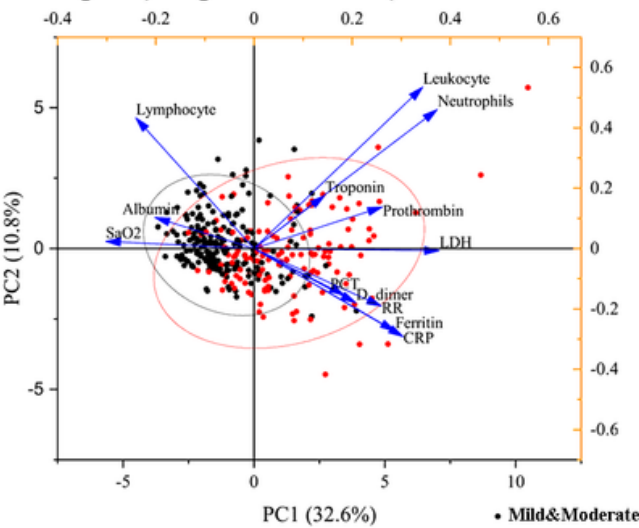

B. Bioplot (End hospitalization)

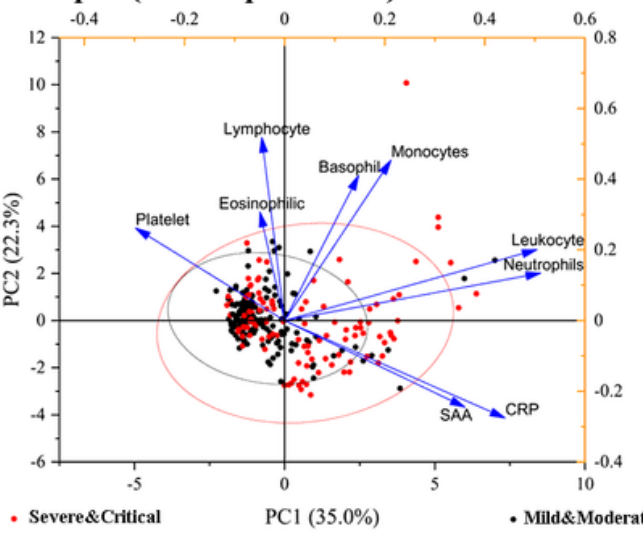

C. Bioplot (Hospital admission)

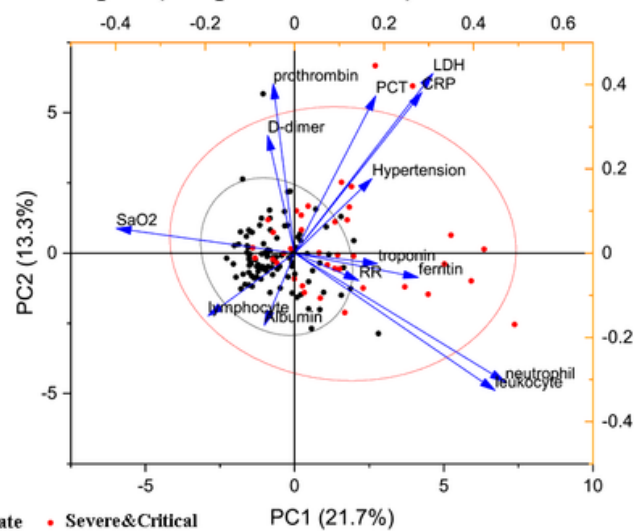

Figure 4

Principal component analysis of variables for explaining proportion to the severity of COVID-19. A) Biplot of indexes expression on hospital admission in the discovery stage; B) Biplot of indexes expression at end hospitalization in the discovery stage; C) Biplot of indexes expression on hospital admission in the validation stage. Samples are shown as dots and colored by outcomes (black indicates mild-to-moderate cases; red represents severe-to-critically ill patients). Indexes shown as lines with arrows. The configuration of indexes on biplot represented relationship between variables and principal components. Each axis represents one principal component (PC), with PC1 on the $x$ axis and PC2 on the $y$ axis.
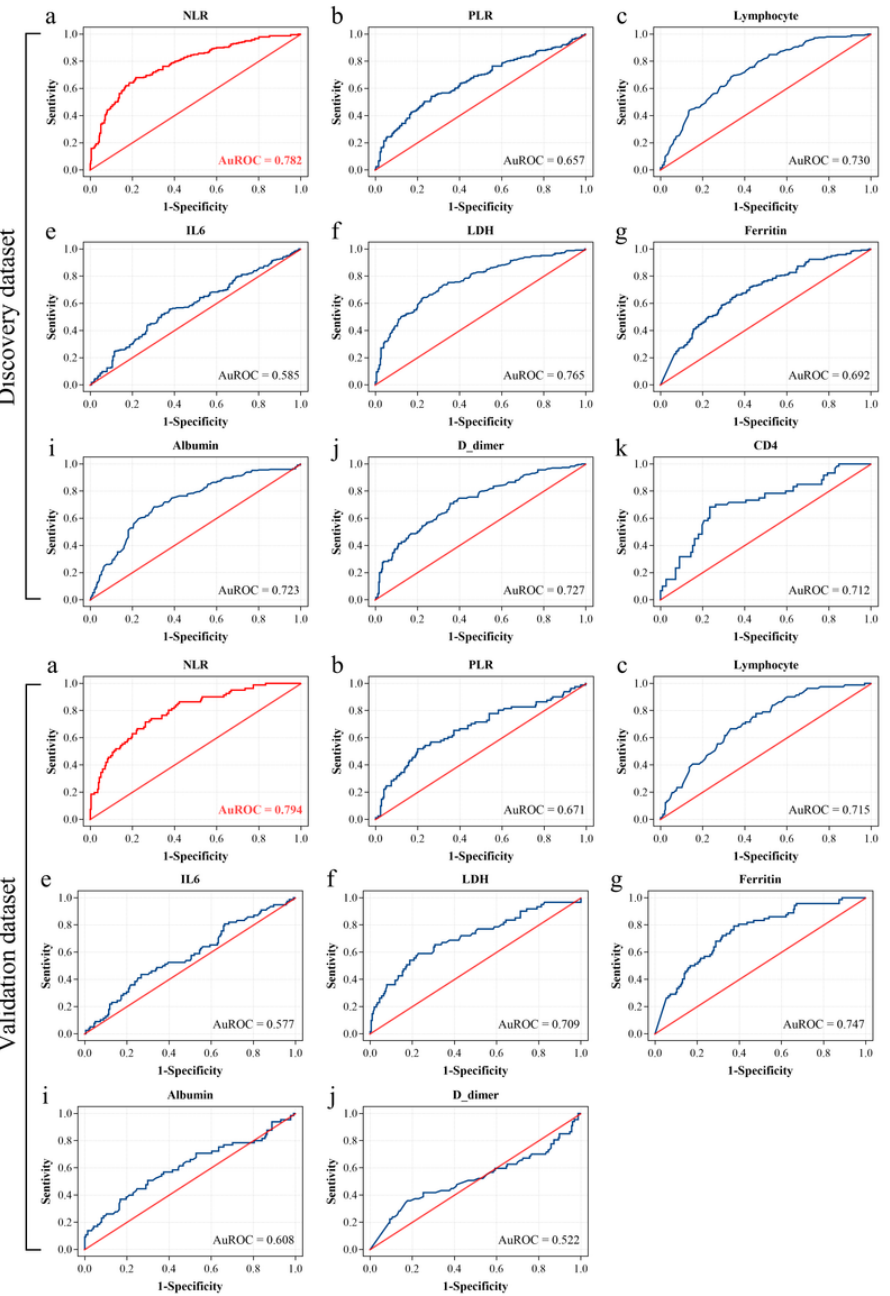
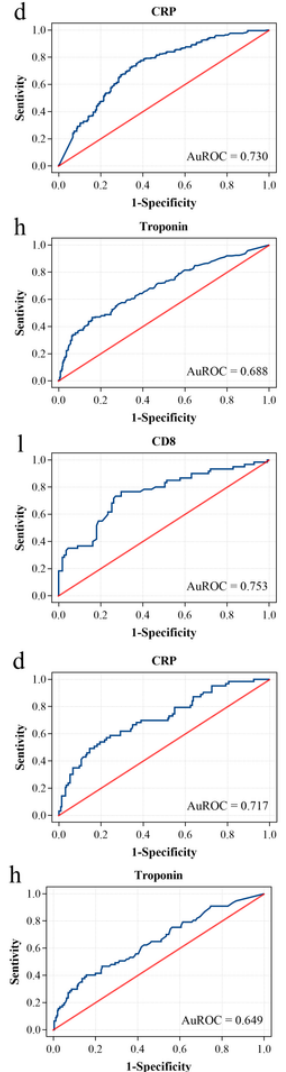
Figure 5

Receiver operating characteristic (ROC) curves of published indexes based on literatures for the severity prediction of COVID-19. ROC curves of NLR, PLR, lymphocyte, CRP, IL-6, LDH, serum ferritin, high-sensitivity cardiac troponin I, albumin, D-dimer, CD4+ T cell and CD8+ T cell for adverse outcome prediction. The area under the curve (AUROC) of NLR, PLR, lymphocyte, CRP, IL-6, LDH, serum ferritin, high-sensitivity cardiac troponin I, albumin, D-dimer, CD4+ T cell and CD8+ $T$ cell was $0.782,0.657,0.730,0.730,0.585,0.765,0.692,0.688,0.723,0.727,0.712$ and 0.753 in the discovery stage (a-I), respectively. It showed that NLR had the biggest AuROC. The area under the curve (AuROC) of NLR, PLR, lymphocyte, CRP, IL-6, LDH, serum ferritin, high-sensitivity cardiac troponin I, albumin and D-dimer was $0.794,0.671,0.715,0.717,0.577,0.709,0.747,0.649,0.608$ and 0.522 in the validation stage (a-j), respectively. NLR still demonstrated to be the biggest AuROC.

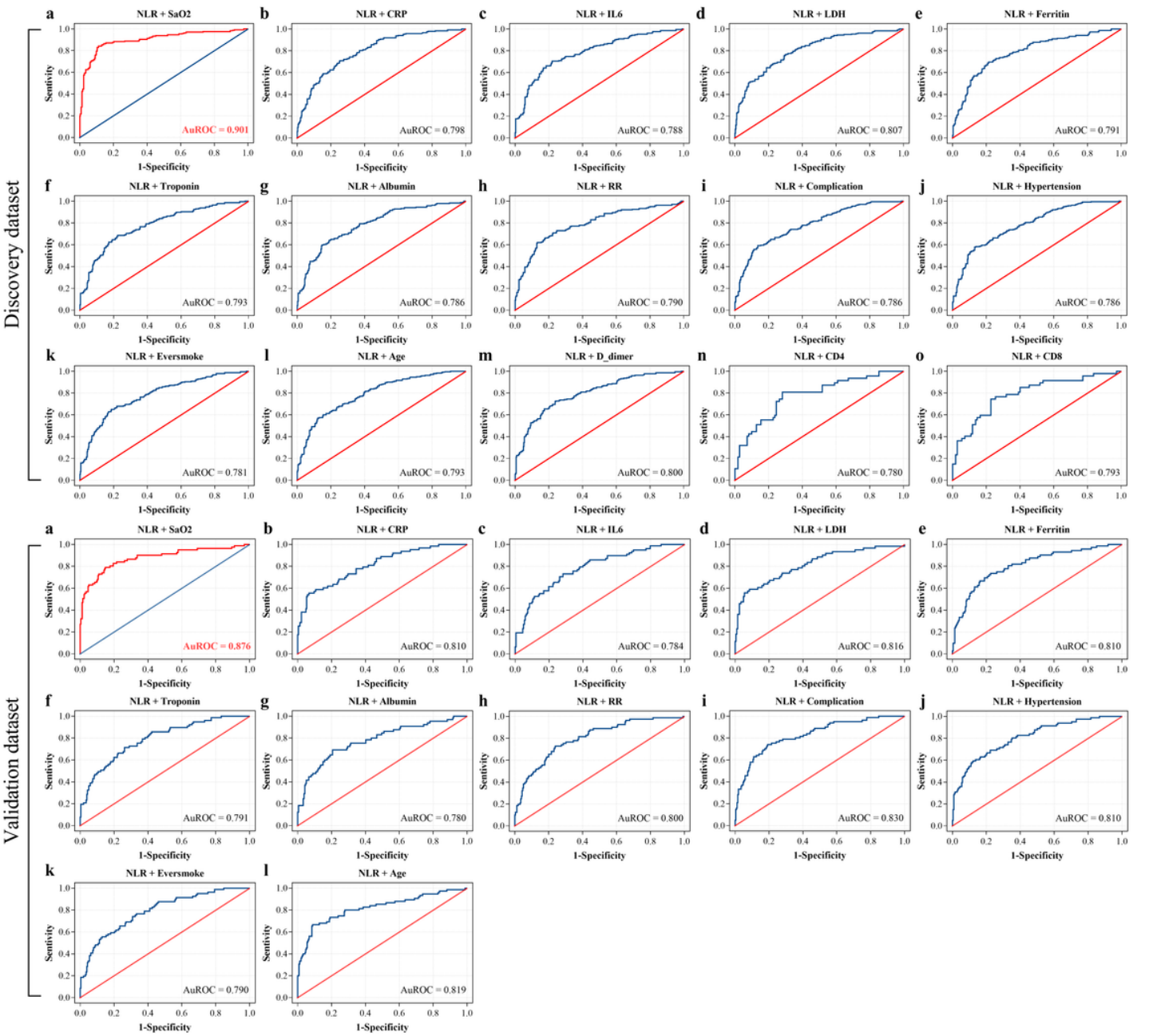

Figure 6

Receiver operating characteristic (ROC) curves of the combination of NLR and another published index based on literatures for the prediction of COVID-19 severity. ROC curves of NLR + CRP, NLR + IL-6, NLR + LDH, NLR + ferritin, NLR + CD4+ T cell, NLR + CD8+ T cell, NLR + high-sensitivity cardiac troponin I, NLR + albumin, NLR + D-dimer, NLR + SaO2, NLR + hypertension, NLR + complication, NLR + RR, NLR + ever smoke and NLR + age for adverse outcome prediction. The area under the curve (AuROC) of NLR + CRP, NLR + IL-6, NLR + LDH, NLR + ferritin, NLR + CD4+ T cell, NLR + CD8+ T cell, NLR + high-sensitivity cardiac troponin I, NLR + albumin, NLR + D-dimer, NLR + SaO2, NLR + hypertension, NLR + complication, NLR + RR, NLR + ever smoke and NLR + age was 0.798, $0.788,0.807,0.791,0.780,0.793,0.793,0.786,0.800,0.901,0.786,0.786,0.790,0.781$ and 0.793 in the discovery stage (a-o), respectively. It showed that NLR + SaO2 had the biggest AuROC. The area under the curve (AuROC) of NLR + SaO2, NLR + CRP, NLR + IL-6, NLR + LDH, NLR + ferritin, NLR + high-sensitivity cardiac troponin I, NLR + albumin, NLR + hypertension, NLR + complication, NLR + RR, NLR + ever smoke and NLR + age was $0.876,0.810,0.784,0.816,0.810$, $0.791,0.780,0.810,0.830,0.800,0.790$ and 0.819 in the validation stage (a-l), respectively. NLR + SaO2 still demonstrated to be the biggest AuROC. 

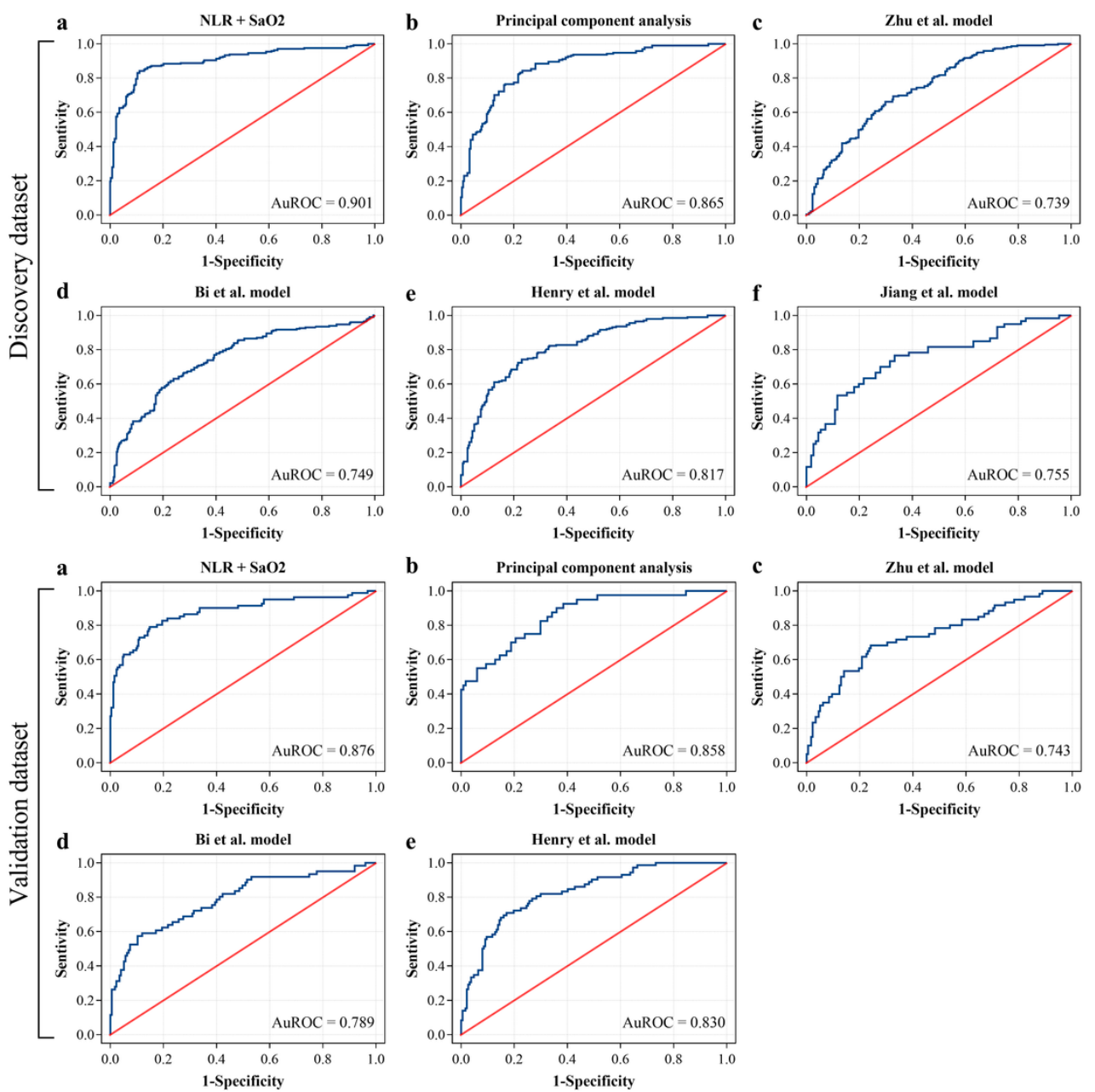

\section{Figure 7}

Receiver operating characteristic (ROC) curves of NLR + SaO2, principal component analysis (PCA) and other published models for the prediction of COVID-19 severity. ROC curves of NLR + SaO2, PCA, Zhu et al. model, Bi et al. model, Henry et al. model and Jiang et al. model for adverse outcome prediction. The area under the curve (AuROC) of NLR + SaO2, PCA, Zhu et al. model, Bi et al. model, Henry et al. model and Jiang et al. model was 0.901, 0.865, 0.739, 0.791, 0.749, 0.817 and 0.755 in the discovery stage (a-f), respectively. It showed that NLR + SaO2 had the biggest AuROC. ROC curves of NLR + SaO2, PCA, Zhu et al. model, Bi et al. model, Henry et al. model and Jiang et al. model for adverse outcome prediction. The area under the curve (AuROC) of NLR + SaO2, PCA, Zhu et al. model, Bi et al. model, Henry et al. model and Jiang et al. model was $0.901,0.865,0.739,0.791,0.749,0.817$ and 0.755 in the validation stage (a-e), respectively. $\mathrm{NLR}+\mathrm{SaO} 2$ still demonstrated to be the biggest AuROC. 

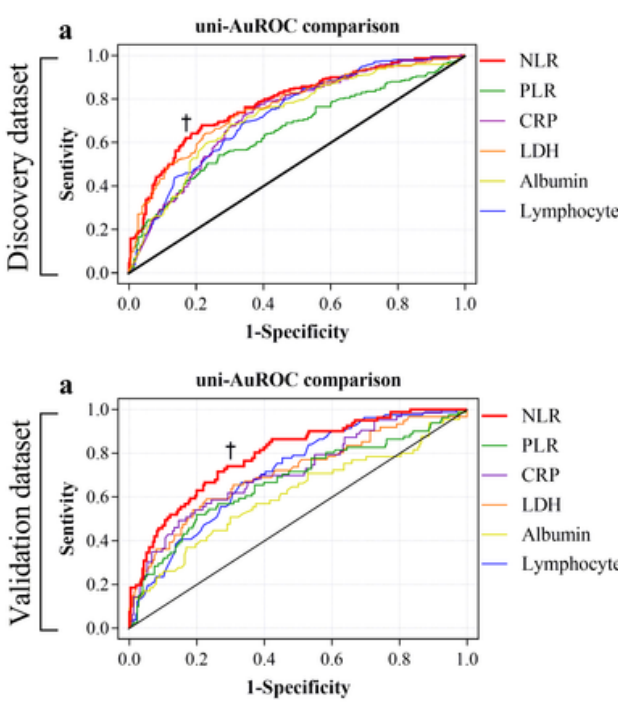

b

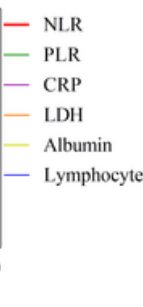

b

b
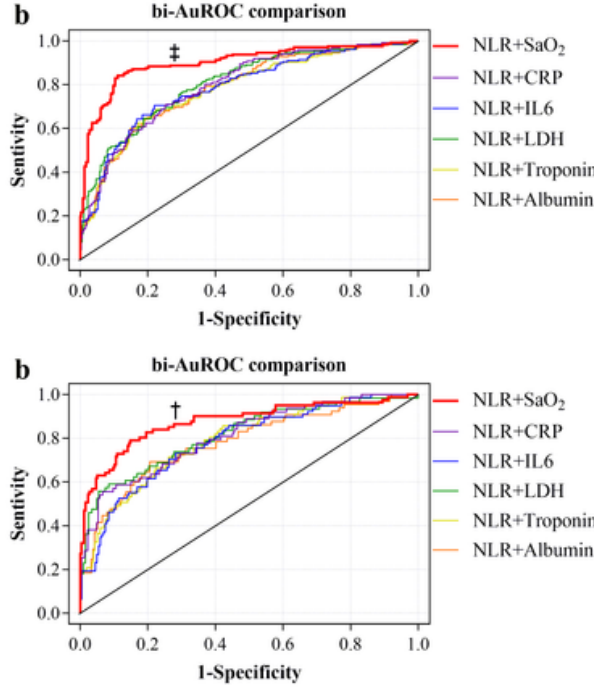

bi-AuROC comparison c

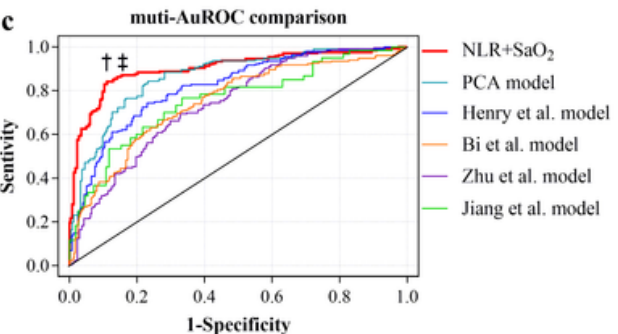

c

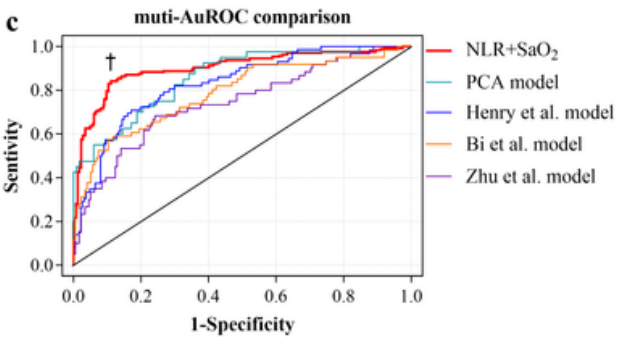

Figure 8

Receiver operating characteristic (ROC) curves of uni-parameter, bi- parameters and muti-parameters for the prediction of COVID-19 severity. The area under the curve (AuROC) of NLR was significantly higher than that of the others among uni-parameters $(p<0.05$, exclude LDH); AuROC of NLR + SaO2 was significantly higher than that of the others both among bi-parameters $(p<0.05)$ and muti-parameters $(p<0.05$, exclude PCA and Henry et al. model) in the discovery stage $(\mathrm{a}-\mathrm{c})$. In the validation stage, AuROC of uni-parameter, bi- parameters and muti-parameters comparisons showed identical trends for the prediction of COVID-19 severity (a-c). 